\title{
Single-cell analysis reveals mechanisms of plasticity of leukemia initiating cells
}

Vivian Morris ${ }^{1}$, William Marion', Travis Hughes ${ }^{2,3}$, Patricia Sousa ${ }^{1}$, Prerana Sensharma ${ }^{1}$, Yana Pikman ${ }^{3,4}$, Marian Harris ${ }^{3,5}$, Alex K. Shalek ${ }^{2}$, Trista E. North ${ }^{1,3}$, George Q. Daley ${ }^{1,3}$, Edroaldo Lummertz da Rocha ${ }^{6+}$, R. Grant Rowe ${ }^{3,7+*}$

${ }^{1}$ Stem Cell Program, Boston Children's Hospital, Boston, MA 02115 USA

${ }^{2}$ Institute for Medical Engineering and Science, Department of Chemistry, and Koch Institute for Integrative Cancer Research, Massachusetts Institute of Technology, Cambridge, MA 02139; Ragon Institute for MGH, MIT, and Harvard, Cambridge, MA 02139; Broad Institute of MIT and Harvard, Cambridge, MA 02142

${ }^{3}$ Harvard Medical School, Boston MA 02115 USA

${ }^{4}$ Department of Pediatric Oncology, Dana-Farber Cancer Institute, Boston, MA 02115 USA

${ }^{5}$ Department of Pathology, Boston Children's Hospital, Boston, MA 02115 USA

${ }^{6}$ Department of Microbiology, Immunology and Parasitology, Federal University of Santa Catarina, Florianópolis, SC, 88040-900, Brazil

${ }^{7}$ Stem Cell Transplantation Program, Boston Children's Hospital, Boston, MA 02115 USA

${ }^{+}$Equal contribution

Number of figures: 4

Number of supplemental figures: 4

Number of supplemental tables: 2

Correspondence

R. Grant Rowe MD PhD

Karp Family Research Building 7

1 Blackfan Circle

Boston MA 02115

Grant rowe@dfci.harvard.edu

Phone: 617-919-6288 


\section{ABSTRACT}

Leukemia initiating cells (LICs) fuel leukemic growth and spark relapse. Previously thought to be primitive and rare, the LIC state may actually be heterogeneous and dynamic, enabling evasion of therapies. Here, we use single-cell transcriptomics to dissect the ontogeny of $M L L-$-rearranged B-lymphoblastic leukemia (MLL-r B-ALL). Although we identify rare transcriptionally and phenotypically primitive LICs, we also observe LICs emerging from more differentiated populations with the capability to replenish the full cellular diversity of $M L L-r$ B-ALL. We find that activation of MYC-driven oxidative phosphorylation controls this process of LIC state conversion. 
Leukemia initiating cells (LICs) possess the capability for sustained, deregulated selfrenewal and the potential to fully reconstitute fulminant leukemia at relapse or upon xenotransplantation. This definition was formed based on the finding that only a specific fraction of human acute myeloid leukemia (AML) cells could engraft immunodeficient mice (Lapidot et al., 1994). Subsequent studies advanced a model wherein leukemia cells are hierarchically organized with rare, primitive, quiescent LICs at the apex that appropriate hallmarks of normal hematopoietic stem and progenitor cells (HSPCs) including self-renewal and differentiation (Kreso and Dick, 2014; Pollyea and Jordan, 2017). Based on this model, LICs have been defined in several forms of leukemia (Aoki et al., 2015; Bonnet and Dick, 1997; Cobaleda et al., 2000; Cox et al., 2004; Kong et al., 2008; Somervaille and Cleary, 2006).

Recent advances have opened paradigms of LIC biology to revision. Use of improved immunodeficient mouse strains to heighten the sensitivity of xenotransplantation has revealed that engraftable LICs can be heterogeneous (Ho et al., 2016; McKenzie et al., 2019; Sarry et al., 2011). This concept could explain the limited clinical success of interventions targeting LICs as has been suggested in solid tumors where cancer stem cells show phenotypic plasticity (Gupta et al., 2019; Pollyea and Jordan, 2017). Although such findings have led to reassessment of classical LIC models, it remains generally accepted that leukemias are comprised of heterogeneous cells with variable xenotransplantation capacities. Since the engraftability of individual leukemias a readout of LIC content - is of prognostic importance, better understanding of LIC biology is needed (Monaco et al., 2004; Paczulla et al., 2017).

B-ALL with rearrangement of the $M L L$ locus (MLL-r) constitutes about $80 \%$ of $\mathrm{B}$ ALL of infancy and also occurs in older children and adults. MLL-r B-ALL behaves aggressively, often presenting with corticosteroid resistance and central nervous system infiltration, and has poor long-term outcomes (Pieters et al., 2019; Pieters et al., 2007). 
This unfavorable clinical behavior is associated with distinctive underlying biology including coexpression of markers of myeloid differentiation and the ability to undergo a B-lymphoid-to-myeloid lineage switch, suggestive of a primitive HSPC of origin and multipotency programs in LICs (Orbach et al., 2013; Pieters et al., 2007; Rossi et al., 2012).

Here, we used single cell RNA sequencing (scRNA-seq) combined with xenotransplantation to define the cellular diversity of MLL-r B-ALL. Although LICs are enriched in phenotypically and transcriptionally primitive fractions of $M L L-r$ B-ALL, they can facultatively emerge from more differentiated blast populations to reconstitute the full cellular diversity of MLL-r B-ALL, a process regulated by MYC signaling and mitochondrial oxidative phosphorylation. Our findings define new mechanisms of LIC plasticity with likely therapeutic relevance. 


\section{RESULTS}

\section{Single cell RNA sequencing identifies candidate LICs}

The coexpression of myeloid markers and tendency to switch to the myeloid lineage at relapse suggests that MLL-r B-ALL LICs possess lympho-myeloid multipotency (Pieters et al., 2007; Rossi et al., 2012). Therefore, we aimed to elicit multipotency at the singlecell level in MLL-r B-ALL. To this end, we employed patient derived xenografts (PDXs) with defined somatic mutations (Table S1)(Kluk et al., 2016; Townsend et al., 2016). These MLL-r B-ALL PDXs infiltrate the liver, spleen, lymph nodes, and central nervous system of unconditioned NOD.Cg-Prkdc ${ }^{\text {scid }} / / 2 r g^{t m 1 W_{j l}}$ (NSG) recipient mice (Figure 1A). At baseline, MLL-r B-ALL cells are nearly uniformly $\mathrm{CD} 19^{+}$with occasional $\mathrm{CD} 33^{+}$cells (Figure 1B). To test for latent multipotency, we used MS5 stromal cells that can induce B-lymphoid and myeloid differentiation in human HSPCs (Doulatov et al., 2010; Issaad et al., 1993). After 4 weeks of culture on MS5, MLL-r B-ALL cells differentiated to CD33 ${ }^{+}$ primitive myeloid cells (Figure S1A). By performing single cell assays, we found that MLL-r B-ALL cells generated clonal outgrowths containing both B-lymphoid and myeloid differentiation (Figure S1B-C).

To better understand the primitive multipotent programs in MLL-r B-ALL, we performed scRNA-seq (Gierahn et al., 2017; Klein et al., 2015). We obtained 5,153 viable, human $\mathrm{CD} 45^{+}$cells from leukemia 1 (patient peripheral blood, inDrop platform) and 6,230 cells from leukemia 2 (early passage PDX, SeqWell platform), after performing quality controls (see Methods). Presenting leukemia 1 (MLL-AF4) as an example, we visualized 14 transcriptional groups with unique signatures using tstochastic neighbor embedding (t-SNE; Figure 1C-D). We used the SingleCellNet algorithm to compare each subpopulation to normal benchmarks and found that most cells classified as pro-B cells, indicating that the observed transcriptional heterogeneity is independent of the global differentiation state (Figure 1E)(Tan and Cahan, 2019; van 
Galen et al., 2019). We next used the StemID algorithm to assign a multipotency label to each cell, finding that clusters 1 and 9 were most highly enriched in putative multipotent cells (Figure 1F)(Grun et al., 2016). Consistent with this prediction, we validated enrichment of primitive HSPC signatures in these clusters (Figure 2G)(Ivanova et al., 2002). We observed similar results in leukemia 2 (Figure S1D-G).

With the aim of enriching LICs, we analyzed the expression of cell surface markers. We found that the primitive HSPC marker CD34 was expressed in the candidate LIC cluster 1; along with the absence of CD38, this suggested a pattern typical of normal human HSCs and multipotent progenitors (MPPs; Figure 1H, Figure S1H)(Doulatov et al., 2012). Given this finding and considering the primitive multipotency programs of MLL-r B-ALL, we analyzed expression of CD34 and CD38 as well as CD90 and CD45RA by flow cytometry. These markers resolve multipotent HSPCs - HSCs, MPPs, and multilymphoid progenitors (MLPs) - from more lineage restricted myeloid progenitors such as granulocyte/monocyte progenitors (GMPs)(Doulatov et al., 2012). We found that $M L L-r$ B-ALL contained three distinguishable populations that we named based on their corresponding normal HSPC immunophenotypes: rare $\mathrm{CD}^{+} 4^{+} \mathrm{CD}^{-} 8^{-} \mathrm{CD}^{-}$

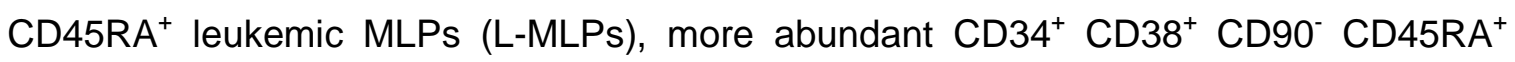
leukemic GMPs (L-GMPs), and the remaining CD34- cells (Figure 2A). Together, these results suggest that MLL-r B-ALL cells are heterogenous with LICs possessing a primitive phenotype and multipotency programs.

\section{MLL-r B-ALL shows functional cellular heterogeneity}

To determine if MLL-r B-ALL LICs are enriched in any of these three populations, we used fluorescence activated cell sorting (FACS) to purify each for analysis (Figure 2B, Figure S1I). Since LICs are typically quiescent, we analyzed the cell cycle status of each population (Guan et al., 2003; Saito et al., 2010; Terpstra et al., 1996). The most 
primitive L-MLP fraction contained the highest proportion of cells in $\mathrm{G}_{0}$ phase (Figure $2 \mathrm{C}$, Figure S2A). Next, we used MS5 assays to quantify leukemic progenitors, finding that LGMPs possessed the highest frequency of short-term clonogenic cells (Figure 2D).

As the gold standard to detect LICs, we next used limiting dilution analysis (LDA) xenotransplantation (Hu and Smyth, 2009). Using the terminal leukemia as our endpoint, we first observed that unfractionated human MLL-r B-ALL possessed a remarkably high frequency of LICs (1/426 cells (1/1417 - 1/128 95\% confidence interval)). Using FACS to fractionate MLL-r B-ALL PDX cells, we found that L-MLPs caused leukemia with the shortest latency and contained the highest LIC content, an effect we corroborated with primary patient cells (Figure 2E-G, Figure S2C-H, Figure S3A-B, Table S2). In line with this finding, using RNA sequencing (RNA-seq) of FACS-purified populations, we found that the L-MLP fraction bore the strongest primitive HSPC signature, paralleling our scRNA-seq data (Figure S3C-D).

Since quiescent LICs may be relatively resistant to chemotherapy, we exposed MLL-r B-ALL cells to prednisolone for five days followed by a washout period to monitor leukemic regeneration, mimicking the corticosteroid prophase used in chemotherapy regimens (Pieters et al., 2007). While most cells died upon prednisolone exposure, we found that L-MLPs were most enriched in the surviving fraction (Figure S3E-G). Over three weeks of culture, we observed that surviving cells underwent a myeloid lineage switch (Figure S3H-J). Together, these results demonstrate that MLL-r B-ALL cells are functionally heterogeneous and appear to be hierarchically organized, with primitive, corticosteroid-resistant, phenotypically plastic, LIC-enriched L-MLPs at the apex.

\section{Plasticity of MLL-r B-ALL LICs}

Although the primitive L-MLP fraction contained abundant LICs, we observed that terminal leukemia did occur in recipients of the more differentiated L-GMP and CD34- 
fractions, albeit at lower efficiency (Figure 2E-G). When we compared the content of terminal leukemia derived from each fraction, we found that the full diversity emerged from each source (Figure 3A). Moreover, by secondary xenotransplantation, we found that leukemia derived from each source was transplantable, demonstrating self-renewal of LICs (Figure 3B). To directly observe this plasticity during leukemic reconstitution in vivo, we transplanted purified CD34- MLL-r B-ALL cells into NSG mice and isolated bone marrow at defined time points post-transplant but prior to the expected onset of terminal leukemia. We found that CD34- cells gradually replenished the CD34+ fraction over time (Figure 3C). To exclude an artifactual effect of the PDX model, we used primary patient blasts and observed similar LIC plasticity in vivo and down to the single-cell level in vitro, where we also observed latent myeloid potential in single-cell clones from all phenotypic populations (Figure S4A-D).

\section{LIC plasticity is driven by MYC and oxidative metabolism}

To investigate mechanisms of LIC plasticity, we performed RNA-seq on relatively LICdeplete CD34- cells either transplanted alone and actively repopulating CD34 ${ }^{+}$ populations or growing at steady state in vivo with L-GMPs and L-MLPs (Figure 4A). Despite their identical immunophenotype, regenerating CD34 cells (CD34 ${ }^{-} \mathrm{r}$ ) and steadystate CD34- cells bore divergent transcriptional profiles (Figure 4B). We found that CD34 $\mathrm{r}$ cells bore signatures of oxidative phosphorylation and MYC target gene expression (Figure 4C). At steady state, these signatures are enriched in multipotent scRNA-seq clusters, suggesting that CD34 $r$ cells are activating primitive programs (Figure 4D). Using available MYC ChIP-seq data, we found that MYC bound loci involved in mitochondrial function and oxidative metabolism (encodeproject.org, Figure 4E). 
Since MYC activity drives mitochondrial oxidative metabolism and is implicated in the pathobiology of MLL-r leukemias, and since LICs rely on oxidative phosphorylation, we investigated the role of this pathway in LIC plasticity (Lagadinou et al., 2013; Lee et al., 2017; Somers et al., 2019). By using the Mitotracker assay, we confirmed that CD34r cells contained higher mitochondrial activity compared to CD34 cells, although overall mitochondrial mass was similar (Figure 4F-G). Next, we generated MV4;11 MLL-r biphenotypic B-ALL/myeloid cells bearing a conditional, doxycycline-inducible MYC expression cassette (Figure 4H)(Lange et al., 1987). Activation of MYC expression was sufficient to increase mitochondrial metabolism (Figure 4I). MYC expression can be inhibited by the bromodomain inhibitor IBET-151 (Dawson et al., 2011). Therefore, we treated MV4;11 cells with IBET-151 and confirmed that this reduced MYC protein (Figure 4J). By using the Seahorse Mito Stress Assay, we found that IBET-151 diminished mitochondrial oxidative metabolism (Figure 4K-L). Next, we treated FACS-sorted CD34r cells in culture on MS5 and actively undergoing regeneration with IBET-151 and monitored state plasticity. We found that IBET-151 inhibited conversion to the CD34 state in culture and diminished active mitochondrial content (Figure 4M-O). Treatment of NSG mice transplanted with CD34-r cells impaired acquisition of a CD34+ phenotype (Figure 4P). These results demonstrate that MYC regulates the metabolic state of $M L L-r$ B-ALL cells as a mechanism of cell state interconversion. 


\section{DISCUSSION}

Since the initial supposition of malignant stem cells, models of leukemic ontogeny have been predicated upon that of normal hematopoiesis (Bonnet and Dick, 1997; Kreso and Dick, 2014; Pollyea and Jordan, 2017). However, the classical model holding that LICs are uniformly primitive akin to normal HSCs have gradually been revised (Pollyea and Jordan, 2017). At first glance, our data would appear to be consistent with this classical model in that MLL-r B-ALL cells are stratified from phenotypically and transcriptionally primitive to most differentiated. However, in B-ALL, LICs are distributed across multiple phenotypic populations, although their relative enrichment in each apparent differentiation state had not been previously quantified (Aoki et al., 2015; Bardini et al., 2015; Kong et al., 2008; Lapidot et al., 1994; le Viseur et al., 2008). Compared to earlier studies, use of more immunodeficient mouse models with heightened sensitivity for human engraftment has uncovered heterogeneity in AML LICs as well ( $\mathrm{Ng}$ et al., 2016; Sarry et al., 2011). Although across many forms of leukemia, LIC ontogeny does not seem to follow the strict stratification and unidirectional differentiation of normal HSPCs, the idea that engraftable LIC content correlates with leukemia prognosis provides an impetus for investigation of the molecular determinants of their state (Monaco et al., 2004; Paczulla et al., 2017). Notably, we find that in MLL-r B-ALL, functional LICs are quite common, being present in approximately $1 / 500$ cells, which is far more frequent than has been reported in limiting dilution of adult AML (Sarry et al., 2011).

Our data reinforce the notion that LIC identity can be fluid. Use of NSG mice led to the first evidence that AML LICs within CD34- fractions could reconstitute the full cellular diversity of the original AML specimen, including more primitive CD34 ${ }^{+}$cells, although the mechanism of this interconversion was not known (Sarry et al., 2011). In a clinical context, in a more recent study, use of matched diagnostic and relapse AML patient specimens revealed that the frequency of engraftable LICs expanded up to 90- 
fold and that LICs gained heterogeneity at relapse, suggestive of plasticity under chemotherapeutic pressure (Ho et al., 2016). Moreover, a recent study reported that acute promyelocytic leukemia cells undergoing differentiation driven by retinoic acid could re-acquire the LIC state following chemotherapy withdrawal, effectively reversing the differentiation trajectory (McKenzie et al., 2019). Extending this concept to solid tumors, a similar effect was recently uncovered in colon cancer, where cells devoid of the stem cell marker Lgr5 can establish metastases and give rise to $\mathrm{Lgr5}^{+}$cancer stem cells (Fumagalli et al., 2020). These findings parallel the known phenomenon that in healthy tissues, adult stem cell identity can be plastic: professional stem cells maintain tissue integrity at steady state, but under stress, facultative stem cells can be recruited from apparently lineage-committed populations (Raven et al., 2017; Tata et al., 2013).

Our efforts to uncover mechanisms of LIC plasticity in MLL-r B-ALL implicated MYC signaling and oxidative phosphorylation. Oncogenic MYC activity is a downstream effector of transforming MLL translocations and mitochondrial turnover is important for LIC homeostasis (Dawson et al., 2011; Pei et al., 2018). Interestingly, MYC can drive mitochondrial oxidative metabolism and proliferation of stem cells in breast cancer, consistent with our findings (Lee et al., 2017). Recruitment of oxidative metabolism seems to also play a role in relapse in B-ALL (Dobson et al., 2020). We impaired MYC activity through inhibition of BET bromodomain proteins - an intervention that slows progression of $M L L$-driven leukemia - finding that this inhibitor blocked the conversion of CD34 $^{-}$to CD34 $^{+}$cells (Bardini et al., 2018; Dawson et al., 2011; Delmore et al., 2011). Together with prior studies, our data support a model wherein MLL-containing translocations cooperate with bromodomain proteins to engage MYC and activate oxidative phosphorylation, driving LIC proliferation and repletion of all leukemic populations. Our findings can be connected to the clinical behavior of MLL-r B-ALL and may have therapeutic implications. The dual lineage and LIC plasticity of MLL-r B-ALL 
provide two distinct mechanisms of chemotherapy evasion. Together with high LIC content, this promiscuity in cell state likely contributes to the poor outcomes of MLL-r BALL. Further understanding of the molecular basis of the enhanced cellular plasticity in MLL-r B-ALL relative to normal hematopoiesis could lead to valuable therapies.

Together, our findings reinforce the notion that LICs are plastic and adaptable, providing possible explanations as to why therapies targeting LICs have yet to prove widespread efficacy despite being the object of intense investigation for over two decades (Pollyea and Jordan, 2017; Saygin et al., 2019). Although aspects of LICs in MLL-r B-ALL might prove to be disease-specific, placement of our results in the broader context of the prevailing knowledge of LICs illustrates the ongoing revision of classical LIC paradigms. Further incremental innovation in immunocompromised mouse models and single-cell-level readouts could continue to augment understanding of LIC biology and define an as yet evasive unifying LIC model. 


\section{ACKNOWLEDGEMENTS}

The authors thank the Single Cell Core at Harvard Medical School, Boston, MA for performing the single-cell RNA-Seq sample preparation. We thank the Molecular Biology Core Facility at the Dana-Farber Cancer Institute for sequencing studies. We thank William Oldham at the Seahorse Core at Brigham and Women's Hospital. We thank the Flow Cytometry Core at Boston Children's Hospital. This work was supported by the National Institute of Diabetes and Digestive and Kidney Diseases (K08 DK114527-01 to R.G.R.) as well as grants from the St. Baldrick's Foundation, Pablove Foundation, and Pedals for Pediatrics (to R.G.R.); and the National Heart, Lung, Blood Institute (U01 HL134812 to G.Q.D.) and the Leukemia and Lymphoma Society of America (to G.Q.D.). We thank Scott Armstrong for helpful discussions.

\section{AUTHOR CONTRIBUTIONS}

Conceptualization, R.G.R and E.L.d.R.; Methodology, R.G.R and E.L.d.R.; Software, E.L.d.R.; Formal analysis, R.G.R and E.L.d.R.; Investigation, V.M., E.L.d.R., W.M., T.H., P.S., P.S., and R.G.R.; Resources, Y.P., M.H., and A.K.S.; Data curation, T.H., R.G.R and E.L.d.R.; Writing - original draft, R.G.R. and E.L.d.R.; Writing - revising and editing, A.K.S., T.E.N., and R.G.R.; Supervision, T.E.N., G.Q.D., and R.G.R.; Project administration, T.E.N., G.Q.D., and R.G.R.; Funding acquisition, T.E.N., G.Q.D., and R.G.R.

\section{DECLARATION OF INTERESTS}

The authors declare no competing interests. 


\section{FIGURE LEGENDS}

Figure. 1. Single cell RNA sequencing identifies candidate LICs.

(A) The indicated tissues were isolated from NSG mice engrafted with human MLL-r B-

ALL PDXs and analyzed by light microscopy (scale $=50 \mu \mathrm{m})$.

(B) (Upper panels) MLL-r B-ALL cells were procured from leukemic NSG bone marrow or spleen and analyzed by flow cytometry (gated on human CD45+). (Lower panels) MLL-r B-ALL cells were examined by light microscopy (scale $=10 \mu \mathrm{m}$; left; leukemia 1; middle leukemia 2; right; leukemia 3).

(C) Human CD45+ MLL-r B-ALL cells were isolated from patient peripheral blood and analyzed by scRNAseq (results shown are for leukemia-1/MLL-AF4). t-distributed stochastic neighbor embedding (t-SNE) was used to visualize populations.

(D) Differentially expressed transcripts in each cluster are shown on a heatmap.

(E) SingleCellNet was used to classify individual cells relative to normal HSPC and differentiated benchmarks.

(F) The StemID algorithm was used to annotate each single cell with a multipotency label, with results overlaid on the t-SNE plot and proportion of predicted multipotent cells in each cluster shown.

(G) t-SNE plot showing enrichment of a validated HSC signature.

(H) The expression of the indicated cell surface markers in each population is shown.

Figure 2. MLL-r B-ALL shows functional cellular heterogeneity.

(A) Representative flow cytometry distributions of cells based on the indicated markers.

(B) The indicated populations were sorted from leukemic marrow (leukemia 1/MLL-AF4) and morphology examined (scale $=10 \mu \mathrm{m})$.

(C) The indicated populations were isolated by FACS and cell cycle state analyzed by flow cytometry following staining for Ki67 and DNA content with DAPI. Cell cycle 
distribution of each population was quantified (results aggregated over two independent experiments, $n=5$ xenografted mice tested; * $p<0.05$ compared to L-MLPs by unpaired student's t-test).

(D) The indicated populations were sorted onto MS5 stromal layers at various doses. After 4 weeks, outgrowths were tabulated, with estimated progenitor cell frequency presented. Results are aggregated over two independent experiments (for L-MLP versus L-GMP $X^{2}=64.3, p=1 \times 10^{-15}$; for L-MLP versus CD34 $X^{2}=14.9, p=0.0001$; for LGMP versus CD34- $\left.\mathrm{X}^{2}=18.1, \mathrm{p}=0.00002\right)$.

(E) 400 cells of the indicated fractions were xenotransplanted into unconditioned NSG recipients, and the incidence of leukemia monitored over time (results aggregated over two independent transplantation experiments; $p=0.008$ L-MLP versus L-GMP, 0.005 LMLP versus CD34; and 0.9 L-GMP versus CD34 compared by log rank test).

(F-G) LIC content of each population was quantified by in vivo LDA with incidence at each dose shown (see Table S2).

\section{Figure 3. Plasticity of MLL-r B-ALL LICs.}

(A) Flow cytometry profiles of terminal leukemias derived from the indicated transplanted cell populations gated on viable and human CD45+ cells within bone marrow. Proportions of each cell type in leukemias derived from the indicated transplanted cell populations are presented ( $p=N S$ comparing each outcome population except in leukemia 1 where $p<0.05$ comparing the L-GMP and CD34 fractions of leukemias derived from L-GMP to both L-MLP and CD34 derived leukemia).

(B) Terminal leukemias derived from the indicated cell sources of leukemia $1 / M L L-A F 4$ in primary transplantation were transplanted into secondary recipients at the indicated doses, and the onset of terminal leukemia was monitored in the secondary recipients. 
(C) CD34 cells were purified from leukemia 1 by FACS and transplanted into recipient mice. Reconstitution of $\mathrm{CD} 34^{+}$populations was monitored by flow cytometry (gated on viable human $\mathrm{CD}_{4} 5^{+}$cells) and quantified over time.

Figure 4. LIC plasticity is driven by MYC and oxidative metabolism.

(A) Schematic showing the input populations sorted for RNA-seq at steady state (left) and from regenerating CD34 cells (right).

(B) RNA-seq was performed on the indicated populations. Representative heatmap of differentially expressed gene is shown across three replicates.

(C) GSEA was used to identify differentially enriched signatures in CD34r versus CD34 cells.

(D) The indicated expression signatures were analyzed in the context of the corresponding scRNA-seq data.

(E) MYC ChIP-seq data from the ENCODE project were analyzed and significantly enriched peaks queried using Gene Ontology analysis, with significant terms presented. (F) Active mitochondria were quantified in the CD34 fraction of bulk leukemia or CD34 $r$ cells using Mitotracker green, with representative flow cytometry results presented compared to background of unstained cells $(n=8$ CD34 and 6 CD34 $r$ biologic replicates over two independent experiments).

(G) CD34 or CD34r cells were processed for transmission electron microscopy (TEM), with representative images presented $($ scale $=1 \mu \mathrm{m})$. Number of mitochondria per cell were quantified over two experiments.

(H) MV4;11 cells were transduced with a doxycycline inducible MYC vector and treated with the indicated doses of doxycycline for 24 hours, at which time MYC was measured by Western blotting. 
(I) MYC expression was induced in MV4;11 cells for 4 days, at which time mitochondrial activity was measured by flow cytometry using Mitotracker ( $n=4$ independent experiments).

(J) MV4;11 cells were treated for 48 hours with 50 nM IBET-151 at which time MYC was measured by Western blot.

(K-L) MV4;11 cells were treated with 50 nM IBET-151 or vehicle control for 48 hours, at which time they were analyzed by the Mito Stress Assay. Plot is representative of five independent experiments analyzed in (L).

(M-O). FACS-sorted CD34 cells were cultured on MS5 stroma for 14 days under the indicated conditions, at which time either the CD34+ content $(\mathrm{M})$ or Mitotracker green signal $(\mathrm{N})$ of the cultures was analyzed by flow cytometry ( $\mathrm{n}=5$ biologic replicates).

(P) CD34-r cells were engrafted into NSG mice for three weeks, at which time a two week treatment course with IBET-151 (5 mg/kg IP daily Monday-Friday for two weeks), after which the human CD45+ content of the bone marrow was analyzed by flow cytometry with the indicated markers ( $n=5$ mice per group).

All results presented as mean \pm SEM and compared by student's $t$-test, with $p$-values for the indicated comparisons shown. 


\section{METHODS}

\section{Study design}

This study was initially designed to define LICs in MLL-r B-ALL, relying on xenotransplantation with LDA and terminal leukemia incidence by day 150 posttransplant as endpoints. This endpoint was chosen based on the approximately 100-day latency of bulk transplanted leukemia cells at a dose of 10,000 cells per transplant. We hypothesized that transplantation of LIC-enriched populations would shift latency earlier and transplantation of LIC-depleted would shift the latency further. Leukemia populations were isolated and xenotransplanted with investigators monitoring recipient mouse health blinded to the cell source. At the earliest onset of discernible morbidity suggestive of active leukemia, mice were humanely euthanized to harvest tissues and bone marrow. At the completion of the experiment, the experimental conditions were unblinded, and LIC frequency calculated by LDA analysis, or survival analyzed by logrank test, where indicated. For quantification of flow cytometry or expression data, the statistical tests used are indicated. Student's t-tests were used and analysis was unpaired, except where otherwise indicated.

\section{Mice and xenotransplantation}

Unconditioned NSG mice (Jackson Laboratory stock 005557) were transplanted with the indicated cell sources at the indicated doses. All transplantation was performed by tail vein injection. Mice transplanted with patient derived xenograft cells or primary patient cells were unconditioned. Mice were followed to the onset of terminal leukemia as indicated in the study design.

\section{Cell culture}


Patient derived leukemia cells were cultured on MS5 stromal cells in the presence of 50 $\mathrm{ng} / \mathrm{ml}$ recombinant human stem cell factor (SCF), $50 \mathrm{ng} / \mathrm{ml}$ recombinant human thrombopoietin (TPO), $10 \mathrm{ng} / \mathrm{ml}$ recombinant human FLT3 ligand (FLT3L), and $10 \mathrm{ng} / \mathrm{ml}$ recombinant human interleukin-7 (IL-7, all from R and D Systems)(Issaad et al., 1993). Leukemia-1 was most amenable to in vitro culture and so was used for most culturebased experiments. For in vitro LDA experiments, 5,000 MS5 cells were plated in wells of gelatin-coated Nunc 96 well plates (Fisher Scientific) with cytokines 48 hours prior to FACS-based sorting of leukemia cells directly into the wells. Where indicated, cells were treated with $50 \mu \mathrm{g} / \mathrm{ml}$ prednisolone (Sigma) for the duration described.

Human MV4;11 cells were cultured in RPMI with $10 \%$ fetal calf serum supplemented with penicillin and streptomycin. Cells were treated with $0.2-2 \mu \mathrm{g} / \mathrm{ml}$ doxycycline hyclate (Sigma) where indicated.

\section{Flow cytometry and cell sorting}

Human antibodies used for flow cytometry studies are listed in the resources table. Data were acquired on either BD LSR Fortessa or LSR II Instruments (BS Biosciences). Cells were sorted on a BD FACS Aria (BD Biosciences) with a $100 \mu \mathrm{m}$ nozzle. Mitotracker Green was purchased from Thermo.

\section{RNA sequencing}

For scRNA-Seq using the Seq-Well platform, 20,000 cells were applied to Seq-Well devices pre-loaded with mRNA capture beads as previously described(Gierahn et al., 2017). Following hybridization and reverse transcription, random second-strand synthesis was performed to generate double stranded cDNA. PCR was performed using the following primer sequence 5' - AAGCAGTGGTATCAACGCAGAGT - 3'. 
Sequencing libraries were generated using the Illumina Nextera XT protocol using custom N700 sequencing indices. Libraries were sequenced using Next-Seq 75 cycle high output sequencing kits with 20 base read 1 sequence and 50 base read 2 sequence.

For inDrops-seq the cells were encapsulated in 2-3 $\mathrm{nl}$ droplets using a microfluidic device and the libraries were made following a previously described protocol(Klein et al., 2015; Zilionis et al., 2017), with the following modifications in the primer sequences. RT primers on hydrogel beads-

5'CGATTGATCAACGTAATACGACTCACTATAGGGTGTCGGGTGCAG[bc1,8nt]GTCT CGTGGGCTCGGAGATGTGTATAAGAGACAG[bc2,8nt]NNNNNNTTTTTTTTTTTTTTTT TTTV- 3'

R1-N6 primer sequence (step 151 in the library prep protocol in (Zilionis et al., 2017))5'TCGTCGGCAGCGTCAGATGTGTATAAGAGACAGNNNNNN-3'

PCR primer sequences (steps 157 and 160 in the library prep protocol in(Zilionis et al., 2017))-

5'-AATGATACGGCGACCACCGAGATCTACACXXXXXXXXTCGTCGGCAGCGTC-3',

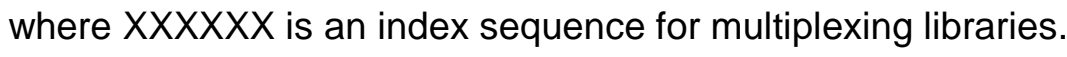

5’- CAAGCAGAAGACGGCATACGAGATGGGTGTCGGGTGCAG-3'

With these modifications in the primer sequences, custom sequencing primers are no longer required. Single-cell RNA-Seq was library preparation was performed by the Single Cell Core at Harvard Medical School, Boston, MA.

\section{Bulk RNA sequencing library prep}


Cells were sorted by FACS and lysed in Trizol reagent (Thermo). RNA was isolated using RNAeasy columns (Qiagen) and low input libraries prepared in collaboration with the Molecular Biology Core at the Dana-Farber Cancer Institute.

\section{Data analysis}

InDrop Single-cell RNA-sequencing

Raw sequencing reads were processed using the inDrop pipeline (https://github.com/indrops/indrops) using default parameters(Klein et al., 2015) The GRCh38 reference genoma was used for alignment of sequencing reads. We used sclmpute to account for dropout rates in single-cell RNA-seq data and obtain an imputed count matrix that was used for all downstream analysis described(Li and Li, 2018). We used sclmpute with the parameter 'Kcluster $=10$ '. To analyze imputed single-cell inDrop data we performed quality control, dimensionality reduction, clustering and differential expression analysis using CellRouter(Lummertz da Rocha et al., 2018). For this leukemia, we applied the following quality control metrics: all genes that were not detected in at least 20 cells were excluded. All cells with less than 200 genes detected were also excluded. As expression of ribosomal or mitochondrial genes is indicative of technical variation in single-cell RNA-seq data we also removed cells where the proportion of the transcript counts derived from mitochondrial genes was greater than $10 \%$ (llicic et al., 2016). After such quality control of the imputed count matrix, we retained 5,153 cells with a median of 15,214 genes detected per cell.

The data was then scaled and used for dimensionality reduction. We performed a principal component (PC) analysis using all genes (34,747 genes) and selected the top 20 PCs using the elbow method. These PCs were used for graph-based clustering to identify clusters of transcriptionally similar cells in our dataset. We also used the top 20 
PCs to perform spectral t-stochastic neighbor embedding (t-SNE) analysis and visualize the underlying cluster structure in a space of reduced dimensionality.

\section{SeqWell single-cell RNA-sequencing}

Read alignment was performed as described(Macosko et al., 2015). Briefly, for each NextSeq sequencing run, raw sequencing data was converted to FASTQ files using bcl2fastq2 that were demultiplexed by Nextera N700 indices corresponding to individual samples. Reads were first aligned to $\mathrm{HgRC} 19$, and individual reads were tagged according to the 12-bp barcode sequence and the 8-bp UMI contained in read 1 of each fragment. Following alignment, reads were binned and collapsed onto 12-bp cell barcodes that corresponded to individual beads using Drop-seq tools (http://mccarrolllab.com/dropseq). Barcodes were collapsed with a single-base error tolerance (Hamming distance $=1$ ), with additional provisions for single insertions or deletions. An identical collapsing scheme (Hamming distance $=1$ ) was then applied to UMls to obtain quantitative counts of individual mRNA molecules. We also used sclmpute to impute the raw counts matrix obtained, with the parameter of 'Kcluster $=6$ '. For this leukemia, we removed all genes expressed in less than 10 cells and also removed all cells expressing less than 500 genes. Cells with transcript counts derived from mitochondrial genes larger than $10 \%$ were also removed. After QC, we retained 6,320 cells with a median of $2,588.5$ genes detected per cell.

\section{Normalization}

Both inDrop and Seq-Well data were analyzed with CellRouter. In CellRouter, transcript counts are normalized using a global scaling normalization method that normalizes expression measurements for each cell by the total expression, multiplied by a scale factor of 10,000, and log-transformed the result. 


\section{StemID analysis}

First, with the raw counts data obtained from the inDrop sample, we performed an initial quality control removing cells not expressing at least 200 genes or genes not expressed in at least 20 cells. This filtered, not normalized counts matrix, was used as input for sclmpute with "Kcluster=10". After data imputation, we used the imputed count matrix for StemID analysis. Briefly, we removed cell cycle genes and performed the StemID analysis setting the following parameters: mintotal $=0.01$, minexpr $=0$, minnumber $=0$, maxexpr=Inf, downsample=TRUE, $d s n=1$ in the "filterdata" function, outminc $=5$, outlg $=2$, probthr $=1 \mathrm{e}-3, \mathrm{thr}=2^{* *}-(1: 40)$, outdistquant $=.95$ in the "findoutliers" function.

\section{SingleCellNet analysis}

We downloaded scRNA-seq data from the GEO accession number GSE116256(van Galen et al., 2019). This study performed a random sampling of hematopoietic cells in the normal and leukemic bone marrow (BM) ecosystem. We reanalyzed five healthy BM samples published with this study and used cell types identified by the authors to train machine learning models of cell type identity of BM cells using SingleCellNet. After training, we classified each single cell in our leukemia samples as belonging to any of the classes in our training dataset.

\section{Signature Scores}

We downloaded gene lists from https://www.gsea-msigdb.org/gsea/msigdb. Specifically, we downloaded the following gene sets: HALLMARK_MYC_TARGETS_V1.txt, HALLMARK_OXIDATIVE_PHOSPHORYLATION.tXt and 
IVANOVA_HEMATOPOIESIS_STEM_CELL.txt. Then, we used CellRouter to calculate signature scores for each cell and plotted the distribution of these scores.

\section{Bulk RNA-sequencing}

Fastq files containing single-end RNASeq reads were aligned with Tophat 2.0.12 against the UCSC hg28 reference genome using Bowtie 2.2.4 with default settings(Kim et al., 2013; Langmead and Salzberg, 2012). Gene level counts were obtained using the subRead featureCounts program (v1.5.1) using the parameter "--primary" and gene models from the UCSC hg28 Illumina iGenomes annotation package(Liao et al., 2014). Read counts were normalized using size factors as available by the DESeq2 package(Love et al., 2014).

\section{Data availability}

The RNA sequencing data acquired in this paper were uploaded to Gene Expression Omnibus under accession number GSE147862.

\section{Morphology}

For morphologic analysis, leukemia cells were spun onto slides and stained with MayGrunwald and Giemsa stains (Sigma) sequentially. Transmission electron microscopy was performed at the electron microscopy core at Harvard Medical School.

\section{Recombinant DNA}

The human MYC cDNA was purchased from Addgene (pDONR223_MYC_WT, a gift from Jesse Boehm and Matthew Meyerson and David Root (plasmid \# 82927; http://n2t.net/addgene:82927 ; RRID: Addgene_82927). The MYC cDNA was cloned into the pCW57.1 vector (gift from David Root (Addgene plasmid \# 41393; 
http://n2t.net/addgene:41393 ; RRID:Addgene_41393) using LR clonase (Thermo). The purified plasmid was used to generate lentivirus in HEK-293T cells, which was used to transduce MV4;11 cells. A stable transduced polyclonal line was selected with $0.5 \mu \mathrm{g} / \mathrm{ml}$ puromycin (Thermo). Gene expression was confirmed by Western blotting following doxycycline exposure

\section{Seahorse Assay}

The Seahorse assays were performed using the Agilent Seahorse XF Cell Mito Stress Test kit using injections of $1 \mu M$ oligomycin, $1 \mu M$ carbonyl cyanide- 4 (trifluoromethoxy) phenylhydrazone (FCCP) and $0.5 \mu \mathrm{M}$ each of antimycin $\mathrm{A}$ and rotenone at the intervals indicated. The instrument is located at the Seahorse Core at Brigham and Women's Hospital.

\section{REFERENCES}

Aoki, Y., Watanabe, T., Saito, Y., Kuroki, Y., Hijikata, A., Takagi, M., Tomizawa, D., Eguchi, M., Eguchi-Ishimae, M., Kaneko, A., et al. (2015). Identification of CD34+ and CD34- leukemia-initiating cells in MLL-rearranged human acute lymphoblastic leukemia. Blood 125, 967-980.

Bardini, M., Trentin, L., Rizzo, F., Vieri, M., Savino, A.M., Garrido Castro, P., Fazio, G., Van Roon, E.H.J., Kerstjens, M., Smithers, N., et al. (2018). Antileukemic Efficacy of BET Inhibitor in a Preclinical Mouse Model of MLL-AF4(+) Infant ALL. Mol Cancer Ther 17, 1705-1716.

Bardini, M., Woll, P.S., Corral, L., Luc, S., Wittmann, L., Ma, Z., Lo Nigro, L., Basso, G., Biondi, A., Cazzaniga, G., et al. (2015). Clonal variegation and dynamic competition of leukemia-initiating cells in infant acute lymphoblastic leukemia with MLL rearrangement. Leukemia 29, 38-50.

Bonnet, D., and Dick, J.E. (1997). Human acute myeloid leukemia is organized as a hierarchy that originates from a primitive hematopoietic cell. Nat Med 3, 730-737. Cobaleda, C., Gutierrez-Cianca, N., Perez-Losada, J., Flores, T., Garcia-Sanz, R., Gonzalez, M., and Sanchez-Garcia, I. (2000). A primitive hematopoietic cell is the target for the leukemic transformation in human philadelphia-positive acute lymphoblastic leukemia. Blood 95, 1007-1013. 
Cox, C.V., Evely, R.S., Oakhill, A., Pamphilon, D.H., Goulden, N.J., and Blair, A. (2004). Characterization of acute lymphoblastic leukemia progenitor cells. Blood 104, 29192925.

Dawson, M.A., Prinjha, R.K., Dittmann, A., Giotopoulos, G., Bantscheff, M., Chan, W.I., Robson, S.C., Chung, C.W., Hopf, C., Savitski, M.M., et al. (2011). Inhibition of BET recruitment to chromatin as an effective treatment for MLL-fusion leukaemia. Nature 478, 529-533.

Delmore, J.E., Issa, G.C., Lemieux, M.E., Rahl, P.B., Shi, J., Jacobs, H.M., Kastritis, E., Gilpatrick, T., Paranal, R.M., Qi, J., et al. (2011). BET bromodomain inhibition as a therapeutic strategy to target c-Myc. Cell 146, 904-917.

Dobson, S.M., Garcia-Prat, L., Vanner, R.J., Wintersinger, J., Waanders, E., Gu, Z., McLeod, J., Gan, O.I., Grandal, I., Payne-Turner, D., et al. (2020). Relapse-Fated Latent Diagnosis Subclones in Acute B Lineage Leukemia Are Drug Tolerant and Possess Distinct Metabolic Programs. Cancer Discov 10, 568-587.

Doulatov, S., Notta, F., Eppert, K., Nguyen, L.T., Ohashi, P.S., and Dick, J.E. (2010). Revised map of the human progenitor hierarchy shows the origin of macrophages and dendritic cells in early lymphoid development. Nat Immunol 11, 585-593.

Doulatov, S., Notta, F., Laurenti, E., and Dick, J.E. (2012). Hematopoiesis: a human perspective. Cell Stem Cell 10, 120-136.

Fumagalli, A., Oost, K.C., Kester, L., Morgner, J., Bornes, L., Bruens, L., Spaargaren, L., Azkanaz, M., Schelfhorst, T., Beerling, E., et al. (2020). Plasticity of Lgr5-Negative Cancer Cells Drives Metastasis in Colorectal Cancer. Cell Stem Cell. Gierahn, T.M., Wadsworth, M.H., 2nd, Hughes, T.K., Bryson, B.D., Butler, A., Satija, R., Fortune, S., Love, J.C., and Shalek, A.K. (2017). Seq-Well: portable, low-cost RNA sequencing of single cells at high throughput. Nat Methods 14, 395-398.

Grun, D., Muraro, M.J., Boisset, J.C., Wiebrands, K., Lyubimova, A., Dharmadhikari, G., van den Born, M., van Es, J., Jansen, E., Clevers, H., et al. (2016). De Novo Prediction of Stem Cell Identity using Single-Cell Transcriptome Data. Cell Stem Cell 19, 266-277. Guan, Y., Gerhard, B., and Hogge, D.E. (2003). Detection, isolation, and stimulation of quiescent primitive leukemic progenitor cells from patients with acute myeloid leukemia (AML). Blood 101, 3142-3149.

Gupta, P.B., Pastushenko, I., Skibinski, A., Blanpain, C., and Kuperwasser, C. (2019). Phenotypic Plasticity: Driver of Cancer Initiation, Progression, and Therapy Resistance. Cell Stem Cell 24, 65-78.

Ho, T.C., LaMere, M., Stevens, B.M., Ashton, J.M., Myers, J.R., O'Dwyer, K.M., Liesveld, J.L., Mendler, J.H., Guzman, M., Morrissette, J.D., et al. (2016). Evolution of acute myelogenous leukemia stem cell properties after treatment and progression. Blood 128, 1671-1678.

Hu, Y., and Smyth, G.K. (2009). ELDA: extreme limiting dilution analysis for comparing depleted and enriched populations in stem cell and other assays. J Immunol Methods 347, 70-78.

Ilicic, T., Kim, J.K., Kolodziejczyk, A.A., Bagger, F.O., McCarthy, D.J., Marioni, J.C., and Teichmann, S.A. (2016). Classification of low quality cells from single-cell RNA-seq data. Genome Biol 17, 29.

Issaad, C., Croisille, L., Katz, A., Vainchenker, W., and Coulombel, L. (1993). A murine stromal cell line allows the proliferation of very primitive human CD34++/CD38progenitor cells in long-term cultures and semisolid assays. Blood 81, 2916-2924. Ivanova, N.B., Dimos, J.T., Schaniel, C., Hackney, J.A., Moore, K.A., and Lemischka, I.R. (2002). A stem cell molecular signature. Science 298, 601-604. 
Kim, D., Pertea, G., Trapnell, C., Pimentel, H., Kelley, R., and Salzberg, S.L. (2013). TopHat2: accurate alignment of transcriptomes in the presence of insertions, deletions and gene fusions. Genome Biol 14, R36.

Klein, A.M., Mazutis, L., Akartuna, I., Tallapragada, N., Veres, A., Li, V., Peshkin, L., Weitz, D.A., and Kirschner, M.W. (2015). Droplet barcoding for single-cell transcriptomics applied to embryonic stem cells. Cell 161, 1187-1201.

Kluk, M.J., Lindsley, R.C., Aster, J.C., Lindeman, N.I., Szeto, D., Hall, D., and Kuo, F.C. (2016). Validation and Implementation of a Custom Next-Generation Sequencing Clinical Assay for Hematologic Malignancies. J Mol Diagn 18, 507-515.

Kong, Y., Yoshida, S., Saito, Y., Doi, T., Nagatoshi, Y., Fukata, M., Saito, N., Yang, S.M., Iwamoto, C., Okamura, J., et al. (2008). CD34+CD38+CD19+ as well as CD34+CD38-CD19+ cells are leukemia-initiating cells with self-renewal capacity in human B-precursor ALL. Leukemia 22, 1207-1213.

Kreso, A., and Dick, J.E. (2014). Evolution of the cancer stem cell model. Cell Stem Cell 14, 275-291.

Lagadinou, E.D., Sach, A., Callahan, K., Rossi, R.M., Neering, S.J., Minhajuddin, M., Ashton, J.M., Pei, S., Grose, V., O'Dwyer, K.M., et al. (2013). BCL-2 inhibition targets oxidative phosphorylation and selectively eradicates quiescent human leukemia stem cells. Cell Stem Cell 12, 329-341.

Lange, B., Valtieri, M., Santoli, D., Caracciolo, D., Mavilio, F., Gemperlein, I., Griffin, C., Emanuel, B., Finan, J., Nowell, P., et al. (1987). Growth factor requirements of childhood acute leukemia: establishment of GM-CSF-dependent cell lines. Blood 70, 192-199.

Langmead, B., and Salzberg, S.L. (2012). Fast gapped-read alignment with Bowtie 2. Nat Methods 9, 357-359.

Lapidot, T., Sirard, C., Vormoor, J., Murdoch, B., Hoang, T., Caceres-Cortes, J., Minden, M., Paterson, B., Caligiuri, M.A., and Dick, J.E. (1994). A cell initiating human acute myeloid leukaemia after transplantation into SCID mice. Nature 367, 645-648.

le Viseur, C., Hotfilder, M., Bomken, S., Wilson, K., Rottgers, S., Schrauder, A., Rosemann, A., Irving, J., Stam, R.W., Shultz, L.D., et al. (2008). In childhood acute lymphoblastic leukemia, blasts at different stages of immunophenotypic maturation have stem cell properties. Cancer Cell 14, 47-58.

Lee, K.M., Giltnane, J.M., Balko, J.M., Schwarz, L.J., Guerrero-Zotano, A.L., Hutchinson, K.E., Nixon, M.J., Estrada, M.V., Sanchez, V., Sanders, M.E., et al. (2017). MYC and MCL1 Cooperatively Promote Chemotherapy-Resistant Breast Cancer Stem Cells via Regulation of Mitochondrial Oxidative Phosphorylation. Cell Metab 26, 633-647 e637. Li, W.V., and Li, J.J. (2018). An accurate and robust imputation method sclmpute for single-cell RNA-seq data. Nat Commun 9, 997.

Liao, Y., Smyth, G.K., and Shi, W. (2014). featureCounts: an efficient general purpose program for assigning sequence reads to genomic features. Bioinformatics 30, 923-930. Love, M.I., Huber, W., and Anders, S. (2014). Moderated estimation of fold change and dispersion for RNA-seq data with DESeq2. Genome Biol 15, 550.

Lummertz da Rocha, E., Rowe, R.G., Lundin, V., Malleshaiah, M., Jha, D.K., Rambo, C.R., Li, H., North, T.E., Collins, J.J., and Daley, G.Q. (2018). Reconstruction of complex single-cell trajectories using CellRouter. Nat Commun 9, 892.

Macosko, E.Z., Basu, A., Satija, R., Nemesh, J., Shekhar, K., Goldman, M., Tirosh, I., Bialas, A.R., Kamitaki, N., Martersteck, E.M., et al. (2015). Highly Parallel Genome-wide Expression Profiling of Individual Cells Using Nanoliter Droplets. Cell 161, 1202-1214. McKenzie, M.D., Ghisi, M., Oxley, E.P., Ngo, S., Cimmino, L., Esnault, C., Liu, R., Salmon, J.M., Bell, C.C., Ahmed, N., et al. (2019). Interconversion between Tumorigenic and Differentiated States in Acute Myeloid Leukemia. Cell Stem Cell 25, 258-272 e259. 
Monaco, G., Konopleva, M., Munsell, M., Leysath, C., Wang, R.Y., Jackson, C.E., Korbling, M., Estey, E., Belmont, J., and Andreeff, M. (2004). Engraftment of acute myeloid leukemia in NOD/SCID mice is independent of CXCR4 and predicts poor patient survival. Stem Cells 22, 188-201.

Ng, S.W., Mitchell, A., Kennedy, J.A., Chen, W.C., McLeod, J., Ibrahimova, N., Arruda, A., Popescu, A., Gupta, V., Schimmer, A.D., et al. (2016). A 17-gene stemness score for rapid determination of risk in acute leukaemia. Nature 540, 433-437.

Orbach, D., Sarnacki, S., Brisse, H.J., Gauthier-Villars, M., Jarreau, P.H., Tsatsaris, V., Baruchel, A., Zerah, M., Seigneur, E., Peuchmaur, M., et al. (2013). Neonatal cancer. Lancet Oncol 14, e609-620.

Paczulla, A.M., Dirnhofer, S., Konantz, M., Medinger, M., Salih, H.R., Rothfelder, K., Tsakiris, D.A., Passweg, J.R., Lundberg, P., and Lengerke, C. (2017). Long-term observation reveals high-frequency engraftment of human acute myeloid leukemia in immunodeficient mice. Haematologica 102, 854-864.

Pei, S., Minhajuddin, M., Adane, B., Khan, N., Stevens, B.M., Mack, S.C., Lai, S., Rich, J.N., Inguva, A., Shannon, K.M., et al. (2018). AMPK/FIS1-Mediated Mitophagy Is Required for Self-Renewal of Human AML Stem Cells. Cell Stem Cell 23, 86-100 e106. Pieters, R., De Lorenzo, P., Ancliffe, P., Aversa, L.A., Brethon, B., Biondi, A., Campbell, M., Escherich, G., Ferster, A., Gardner, R.A., et al. (2019). Outcome of Infants Younger Than 1 Year With Acute Lymphoblastic Leukemia Treated With the Interfant-06 Protocol: Results From an International Phase III Randomized Study. J Clin Oncol 37, 2246-2256. Pieters, R., Schrappe, M., De Lorenzo, P., Hann, I., De Rossi, G., Felice, M., Hovi, L., LeBlanc, T., Szczepanski, T., Ferster, A., et al. (2007). A treatment protocol for infants younger than 1 year with acute lymphoblastic leukaemia (Interfant-99): an observational study and a multicentre randomised trial. Lancet 370, 240-250.

Pollyea, D.A., and Jordan, C.T. (2017). Therapeutic targeting of acute myeloid leukemia stem cells. Blood 129, 1627-1635.

Raven, A., Lu, W.Y., Man, T.Y., Ferreira-Gonzalez, S., O'Duibhir, E., Dwyer, B.J., Thomson, J.P., Meehan, R.R., Bogorad, R., Koteliansky, V., et al. (2017).

Cholangiocytes act as facultative liver stem cells during impaired hepatocyte regeneration. Nature 547, 350-354.

Rossi, J.G., Bernasconi, A.R., Alonso, C.N., Rubio, P.L., Gallego, M.S., Carrara, C.A., Guitter, M.R., Eberle, S.E., Cocce, M., Zubizarreta, P.A., et al. (2012). Lineage switch in childhood acute leukemia: an unusual event with poor outcome. Am J Hematol 87, 890897.

Saito, Y., Uchida, N., Tanaka, S., Suzuki, N., Tomizawa-Murasawa, M., Sone, A., Najima, Y., Takagi, S., Aoki, Y., Wake, A., et al. (2010). Induction of cell cycle entry eliminates human leukemia stem cells in a mouse model of AML. Nat Biotechnol 28, 275-280.

Sarry, J.E., Murphy, K., Perry, R., Sanchez, P.V., Secreto, A., Keefer, C., Swider, C.R., Strzelecki, A.C., Cavelier, C., Recher, C., et al. (2011). Human acute myelogenous leukemia stem cells are rare and heterogeneous when assayed in NOD/SCID/IL2Rgammac-deficient mice. J Clin Invest 121, 384-395.

Saygin, C., Matei, D., Majeti, R., Reizes, O., and Lathia, J.D. (2019). Targeting Cancer Stemness in the Clinic: From Hype to Hope. Cell Stem Cell 24, 25-40.

Somers, K., Wen, V.W., Middlemiss, S.M.C., Osborne, B., Forgham, H., Jung, M., Karsa, M., Clifton, M., Bongers, A., Gao, J., et al. (2019). A novel small molecule that kills a subset of MLL-rearranged leukemia cells by inducing mitochondrial dysfunction. Oncogene 38, 3824-3842. 
Somervaille, T.C., and Cleary, M.L. (2006). Identification and characterization of leukemia stem cells in murine MLL-AF9 acute myeloid leukemia. Cancer Cell 10, 257268.

Tan, Y., and Cahan, P. (2019). SingleCellNet: A Computational Tool to Classify Single Cell RNA-Seq Data Across Platforms and Across Species. Cell Syst 9, 207-213 e202.

Tata, P.R., Mou, H., Pardo-Saganta, A., Zhao, R., Prabhu, M., Law, B.M., Vinarsky, V., Cho, J.L., Breton, S., Sahay, A., et al. (2013). Dedifferentiation of committed epithelial cells into stem cells in vivo. Nature 503, 218-223.

Terpstra, W., Ploemacher, R.E., Prins, A., van Lom, K., Pouwels, K., Wognum, A.W., Wagemaker, G., Lowenberg, B., and Wielenga, J.J. (1996). Fluorouracil selectively spares acute myeloid leukemia cells with long-term growth abilities in immunodeficient mice and in culture. Blood 88, 1944-1950.

Townsend, E.C., Murakami, M.A., Christodoulou, A., Christie, A.L., Koster, J., DeSouza, T.A., Morgan, E.A., Kallgren, S.P., Liu, H., Wu, S.C., et al. (2016). The Public Repository of Xenografts Enables Discovery and Randomized Phase II-like Trials in Mice. Cancer Cell 29, 574-586.

van Galen, P., Hovestadt, V., Wadsworth li, M.H., Hughes, T.K., Griffin, G.K., Battaglia, S., Verga, J.A., Stephansky, J., Pastika, T.J., Lombardi Story, J., et al. (2019). SingleCell RNA-Seq Reveals AML Hierarchies Relevant to Disease Progression and Immunity. Cell 176, 1265-1281 e1224.

Zilionis, R., Nainys, J., Veres, A., Savova, V., Zemmour, D., Klein, A.M., and Mazutis, L. (2017). Single-cell barcoding and sequencing using droplet microfluidics. Nat Protoc 12, 44-73. 
A

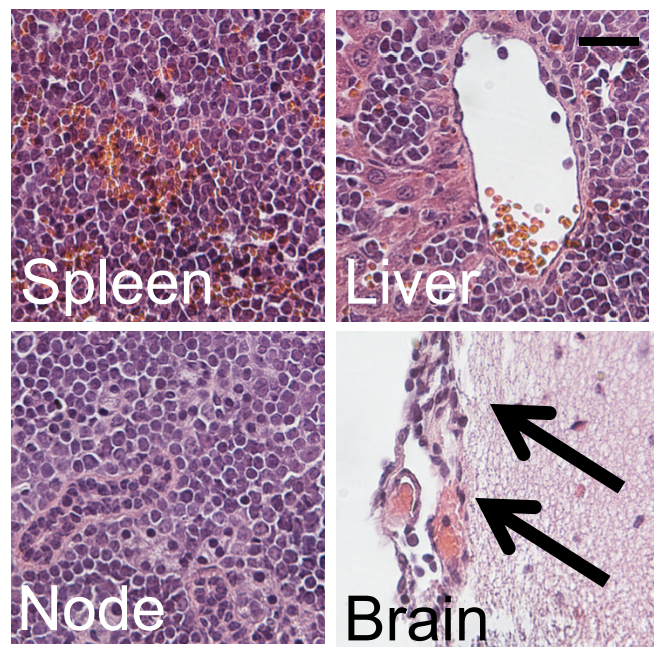

B
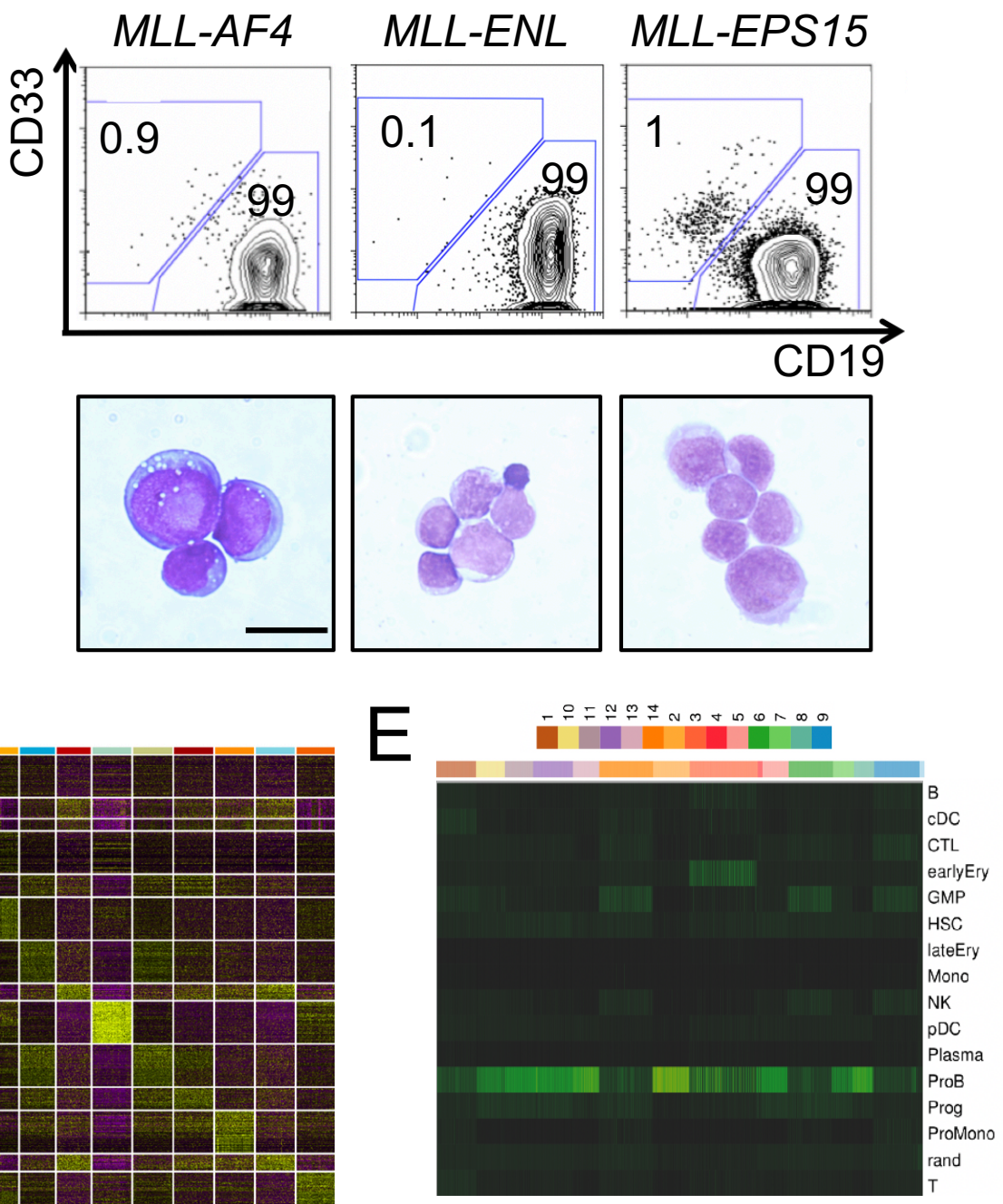

Classification probability

- Multipotent

- Other
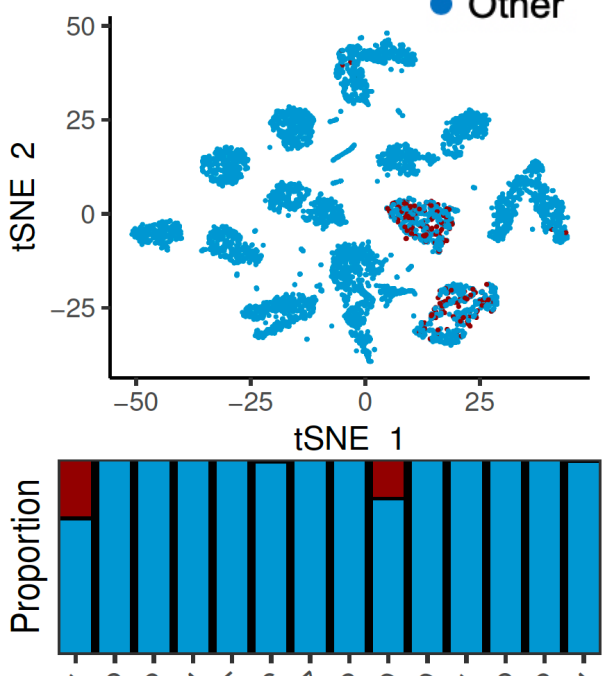

Cluster
Scaled expression $\quad \mid-\infty m \nabla \infty$ $\overrightarrow{\vec{u}}-\dot{\mathrm{i}} \circ \mathrm{O}-\overrightarrow{\mathrm{in}}$

G

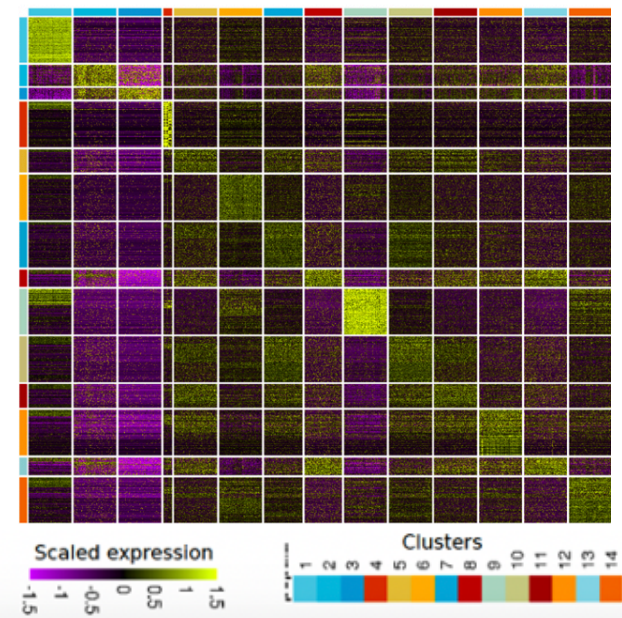

Ivanova HSPC

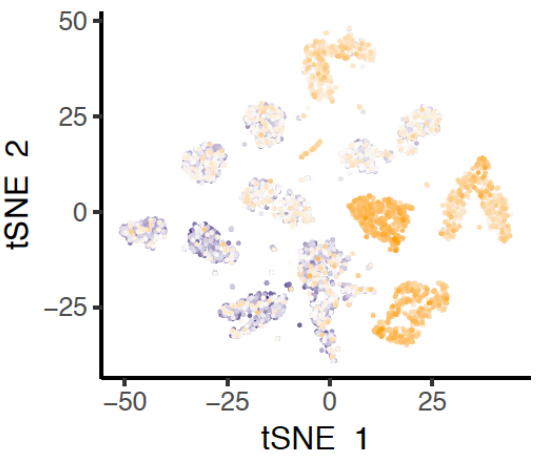

$\begin{array}{lllll}-0.2 & -0.1 & 0.0 & 0.1 & 0.2\end{array}$

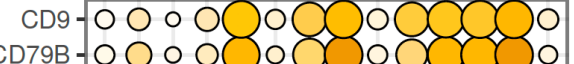

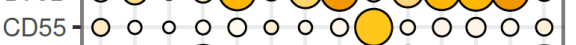
CD63 - O o o 9000900008

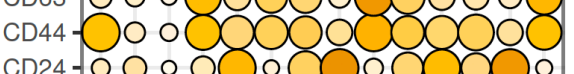
CD24 - 0 O 00000000000000

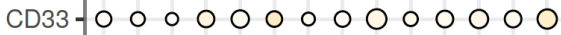
CD19-0 o ० 00000000000

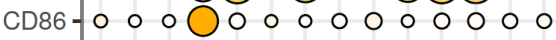
CD226 - 0 ○ 0000000000000

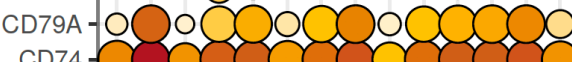

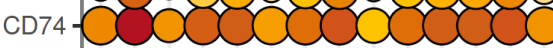

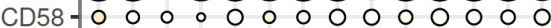
CD46-Oo o 00000000000

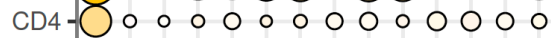

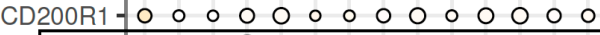
CD38-O O o 00000000000 CD34-0 0000000000000

Average expression

Percentage $0 \bigcirc 00$. $\overrightarrow{8}$ जै जु 12 $3 \times 56189,0,1,2,3, x$ 
A

$\stackrel{\infty}{0}$

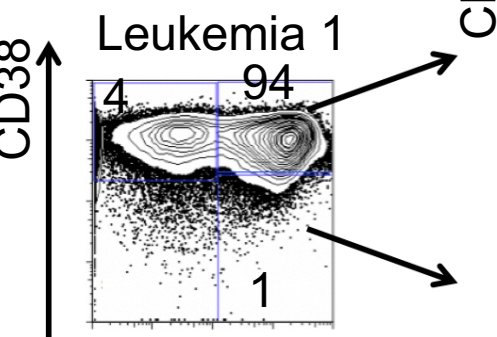

Leukemia 2

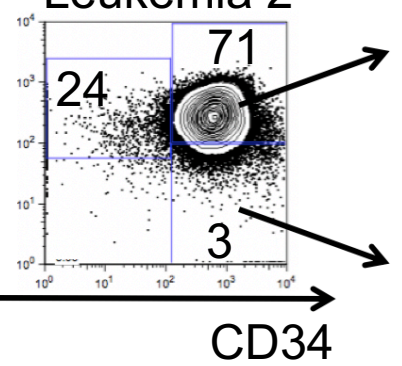

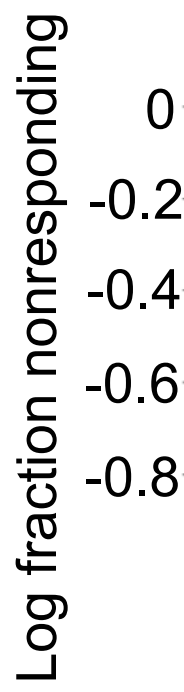

$\mathrm{F}$
B

Figure 2

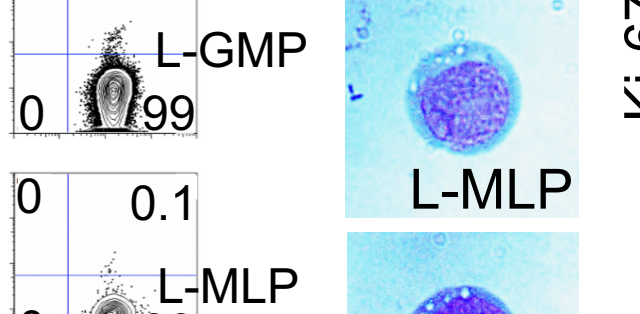

Oิ L-MLP L-GMP CD34-

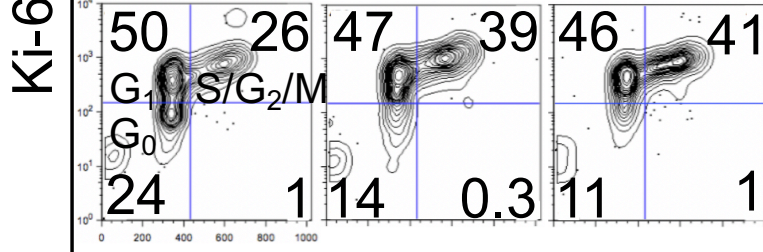

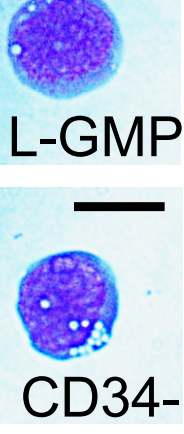

\begin{tabular}{l|l}
0 & 0.1
\end{tabular}

0.099

CD45RA

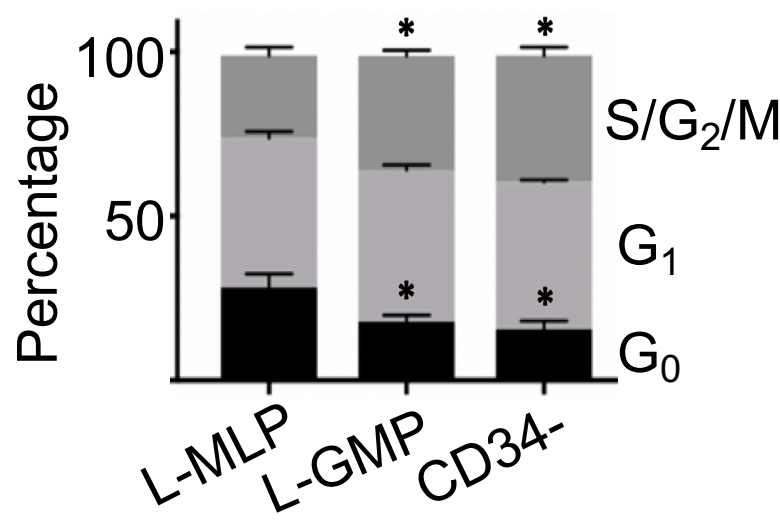

L-MLP 1/50

$(1 / 90-1 / 28)$

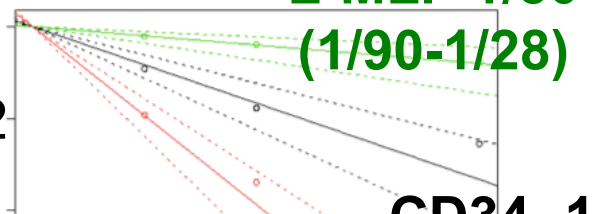

L-GMP 1/5

$(1 / 16-1 / 9)$

$\begin{array}{lllll}0 & 1 & 2 & 3 & 4\end{array}$

Dose (cells)

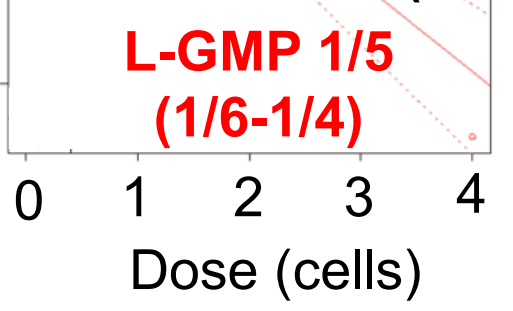

E

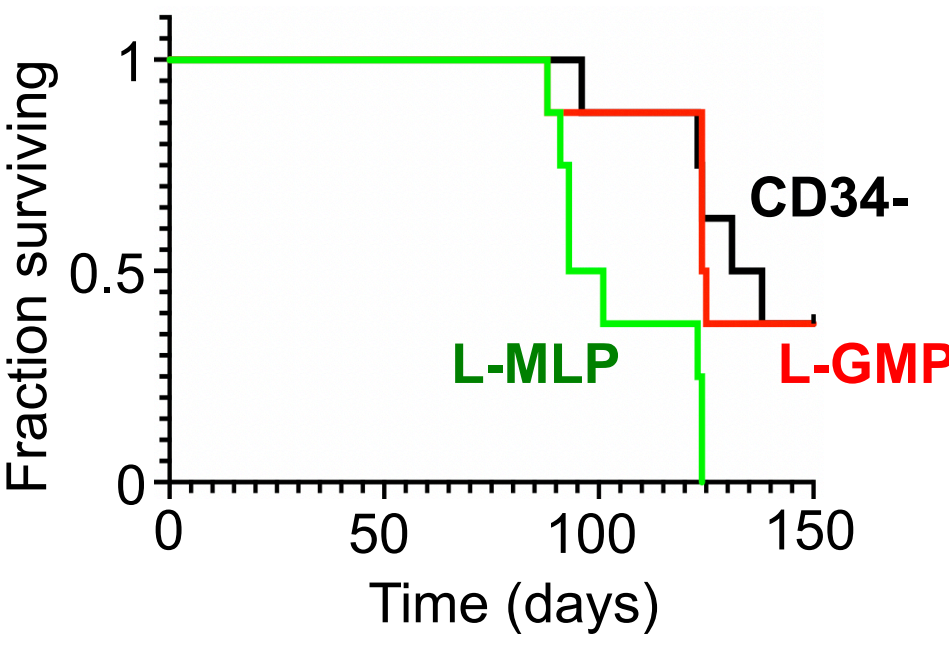

$\mathrm{G}$ 旁

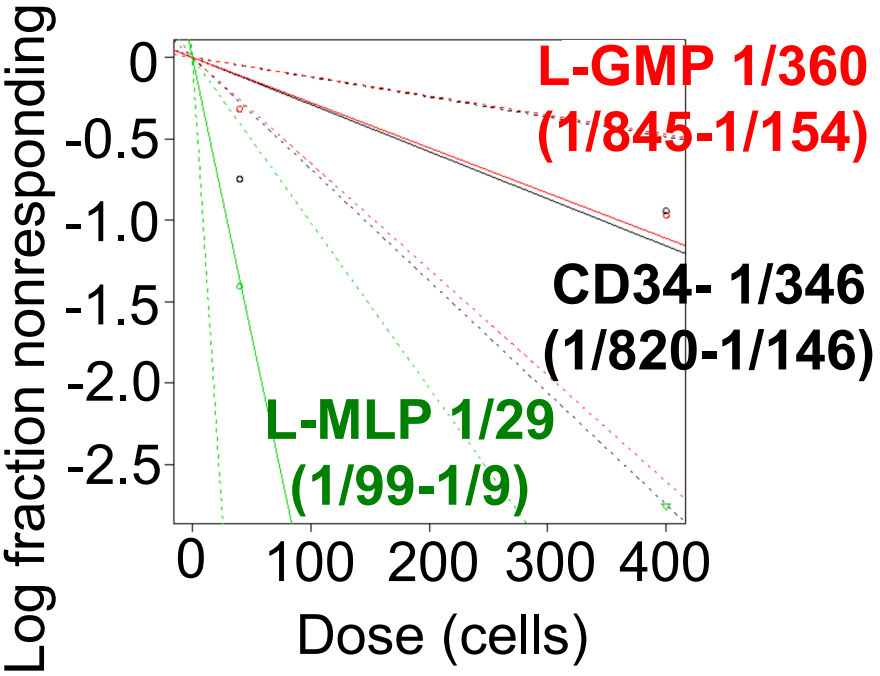


Figure 3
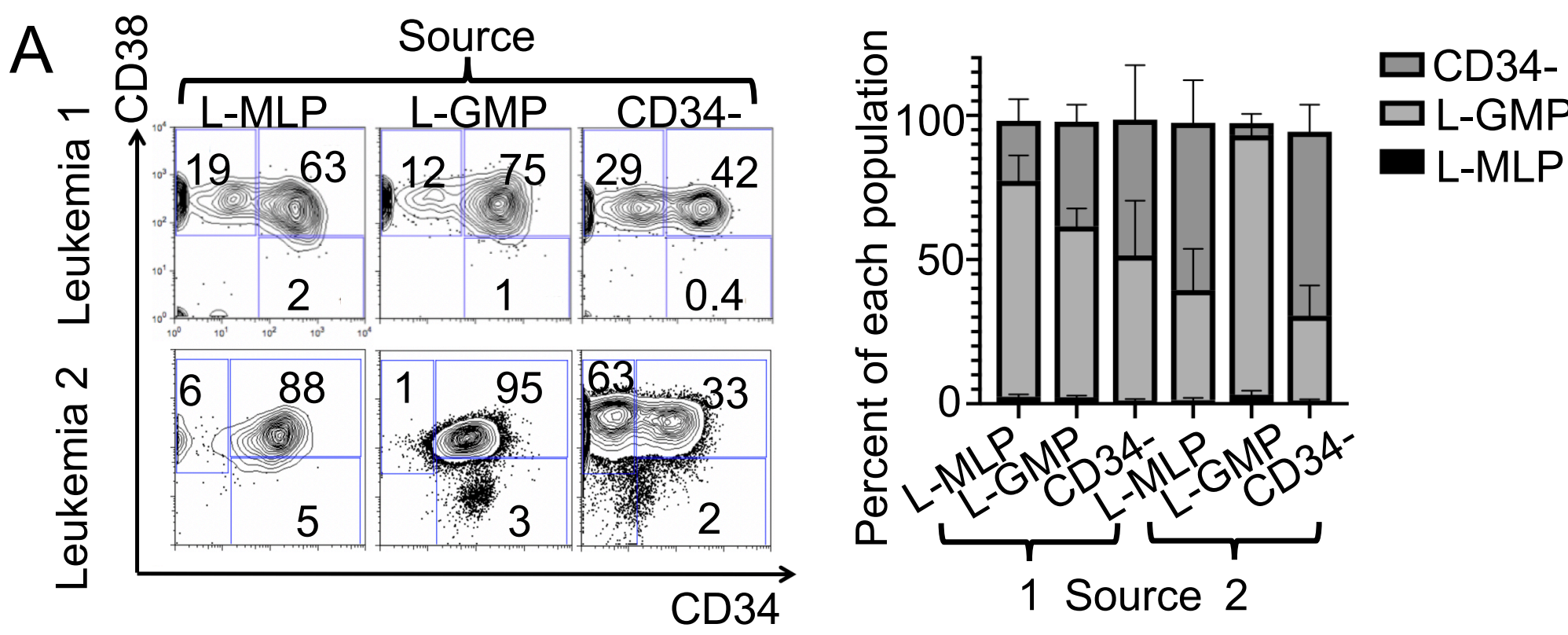

B
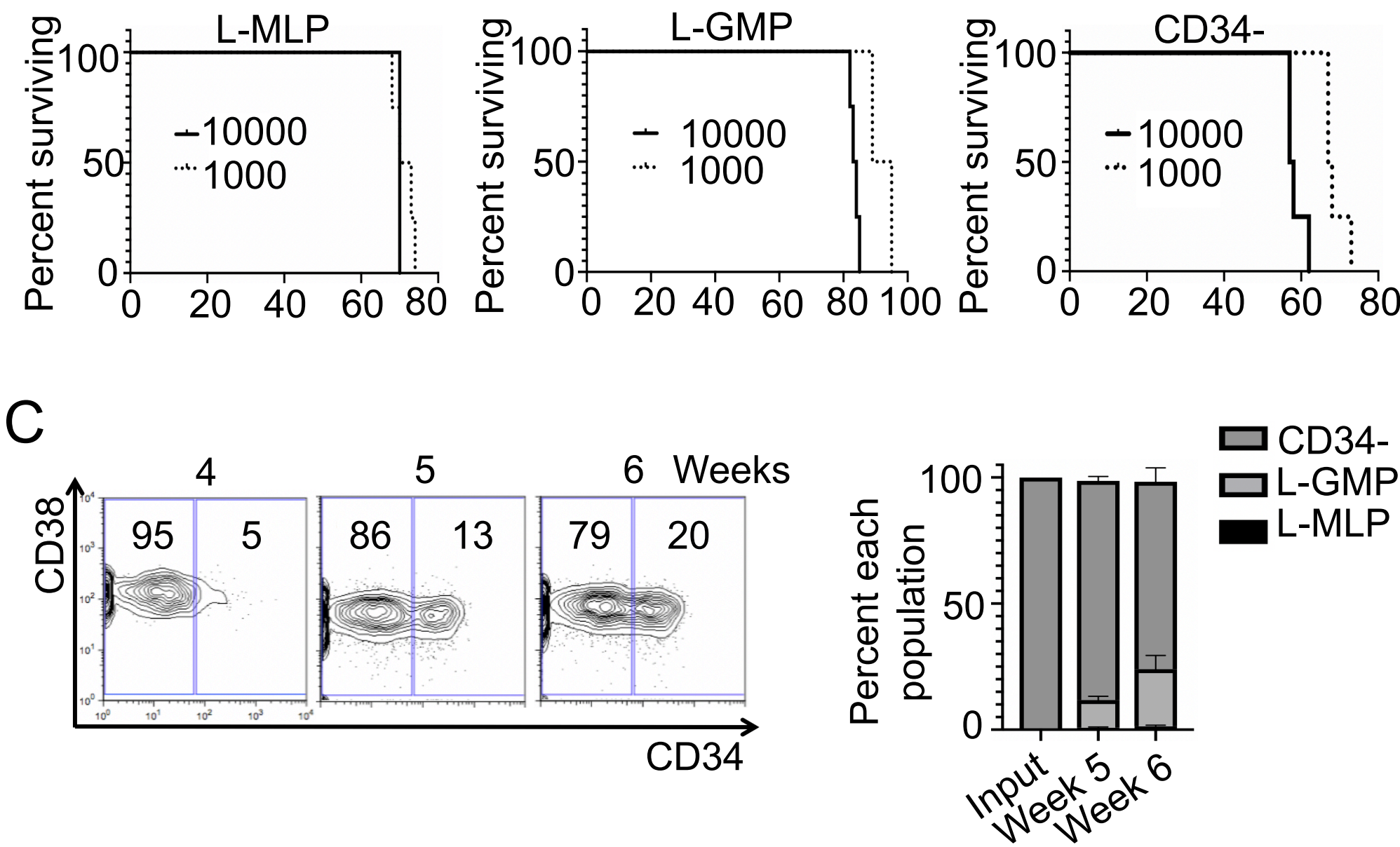

$\square$ CD34口L-GMP L-MLP 
A
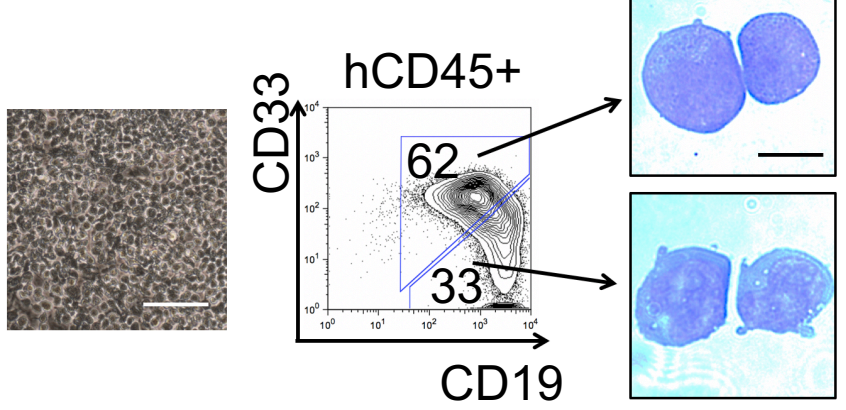

B
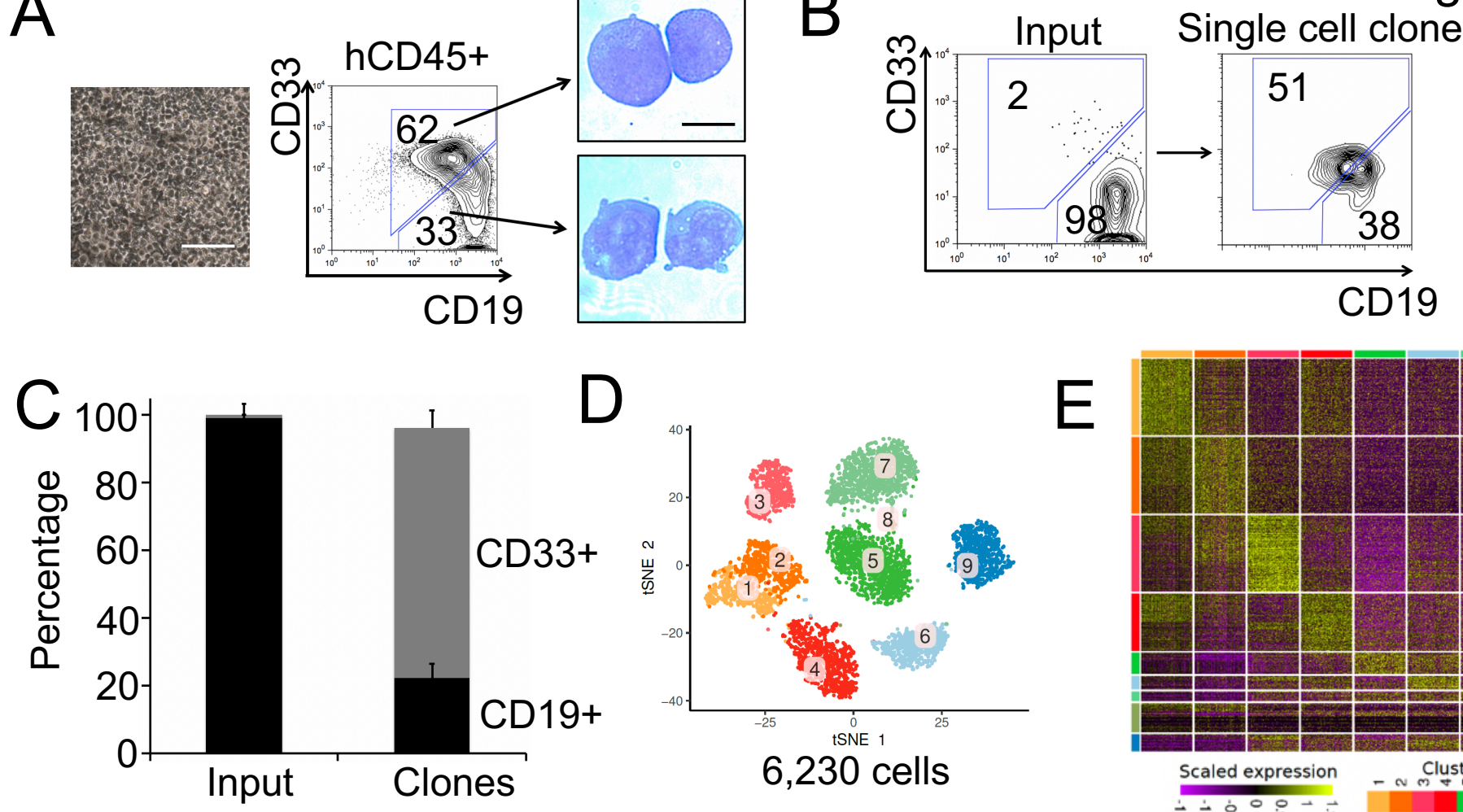

$F$
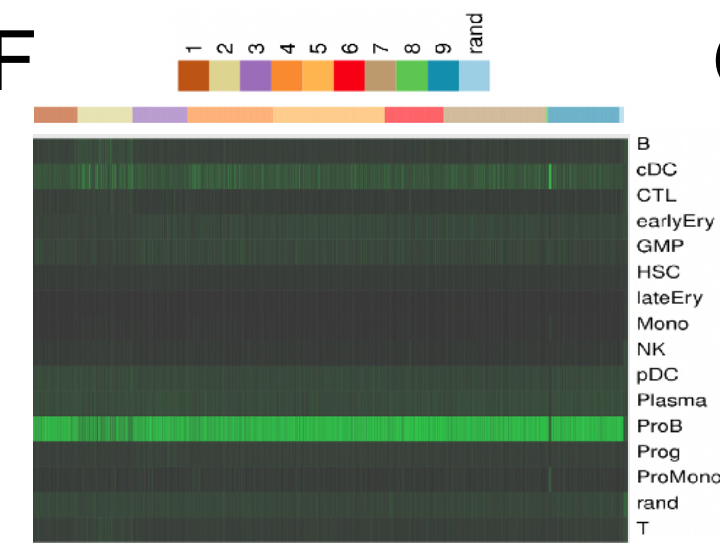

Classification probability

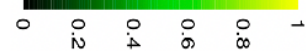

$\mathrm{H}$

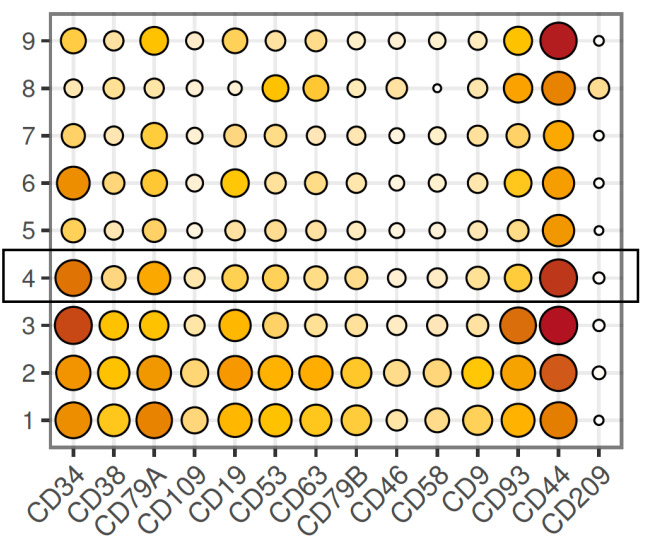

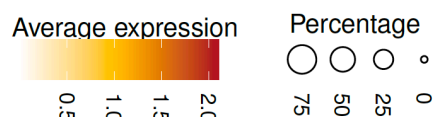

G
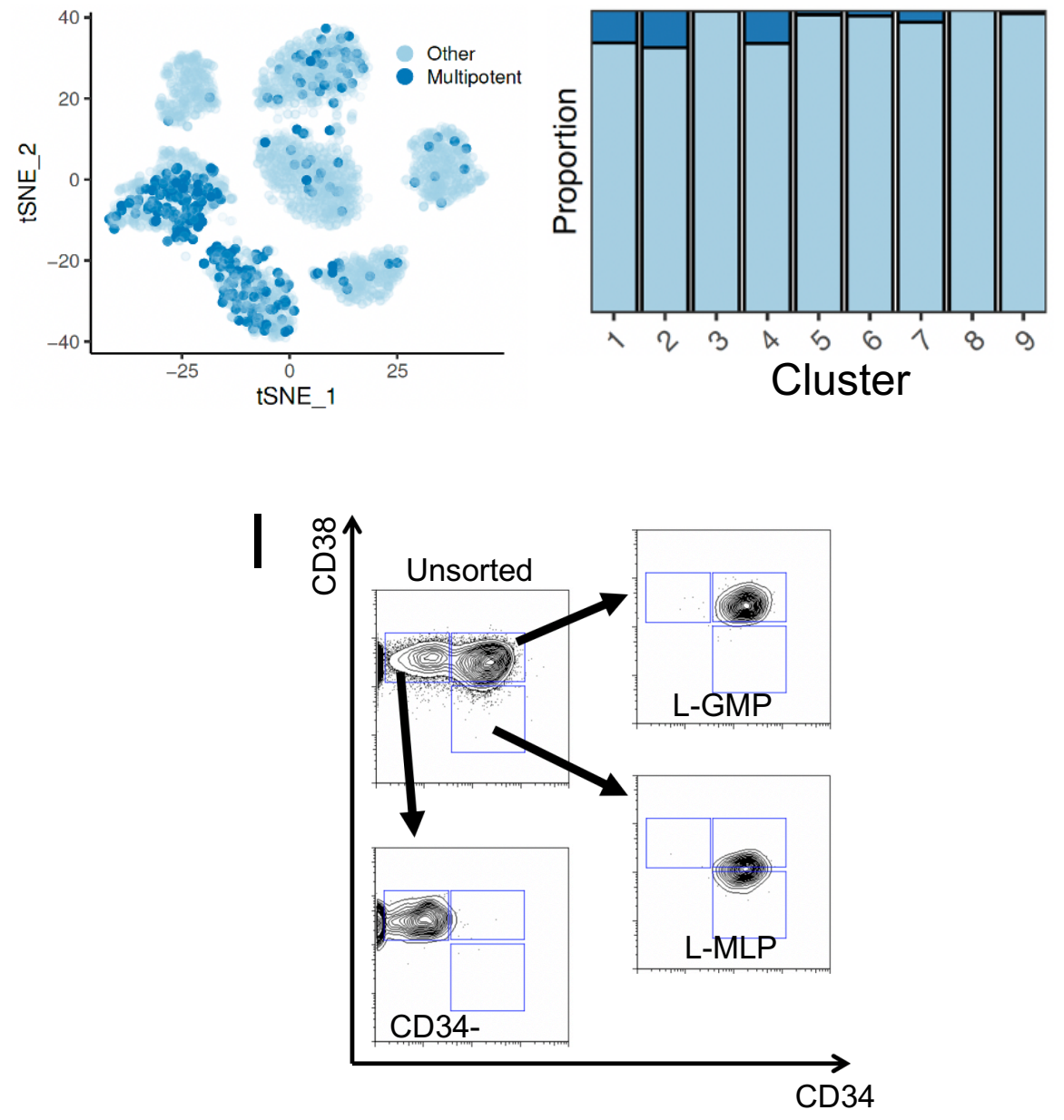


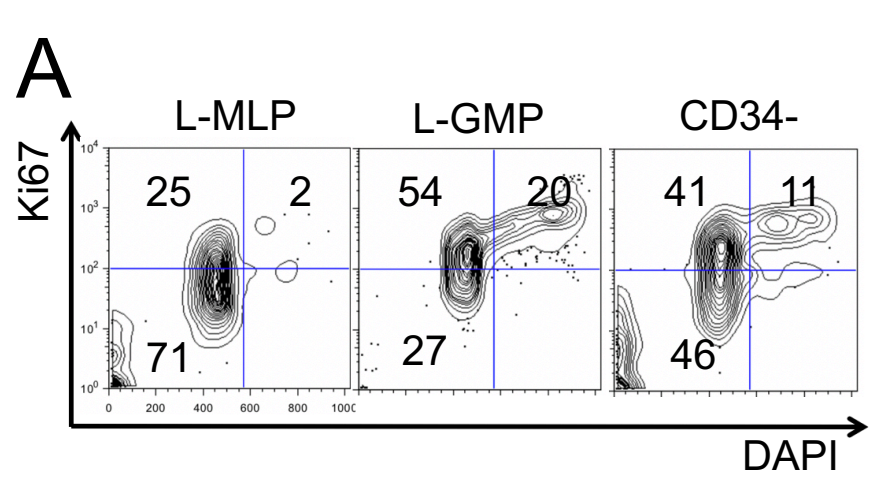

L-MLP

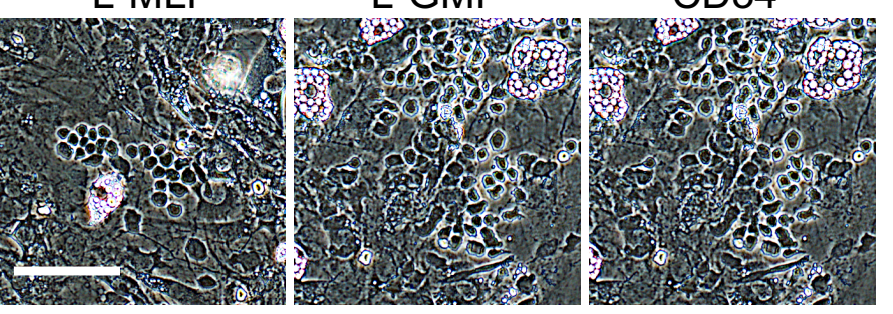

D
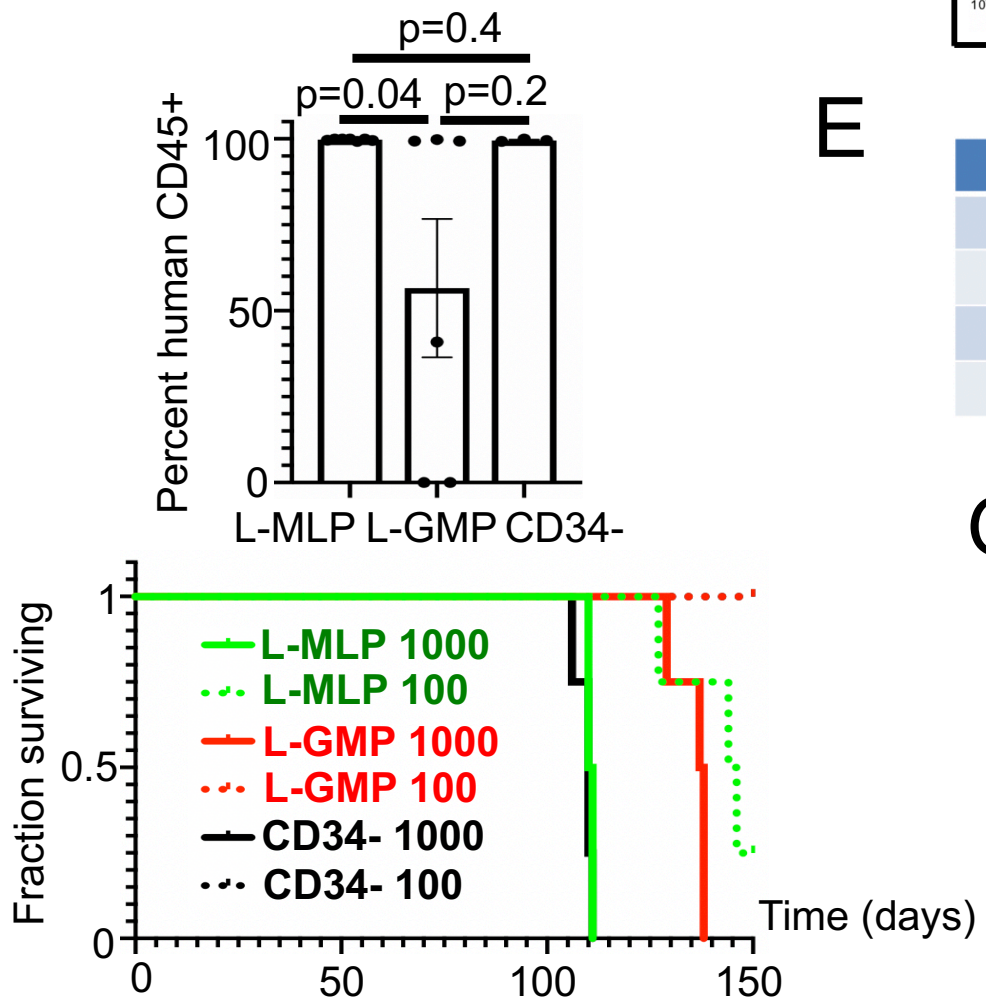

H

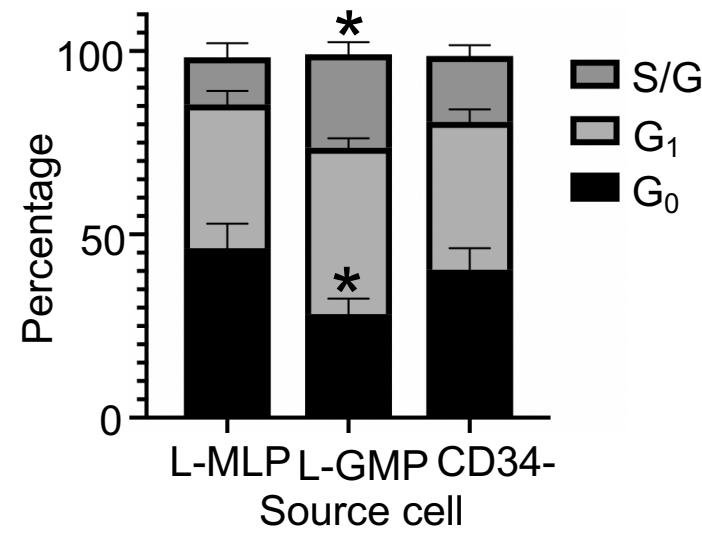

Figure S2

Source cell

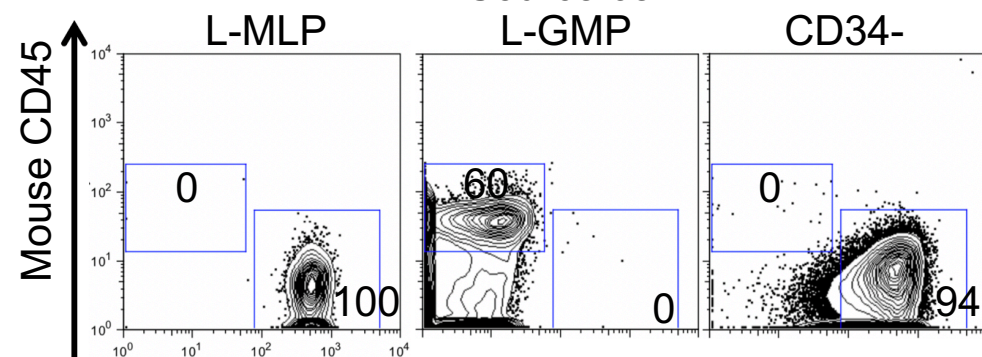

Human CD45

\begin{tabular}{|c|c|c|c|}
\hline Dose & L-MLP & L-GMP & CD34- \\
\hline 4000 & $4 / 4$ & $4 / 4$ & $4 / 4$ \\
\hline 1000 & $4 / 4$ & $4 / 4$ & $4 / 4$ \\
\hline 400 & $2 / 4$ & $1 / 4$ & $2 / 4$ \\
\hline 100 & $3 / 4$ & $0 / 3$ & $0 / 4$ \\
\hline
\end{tabular}

G

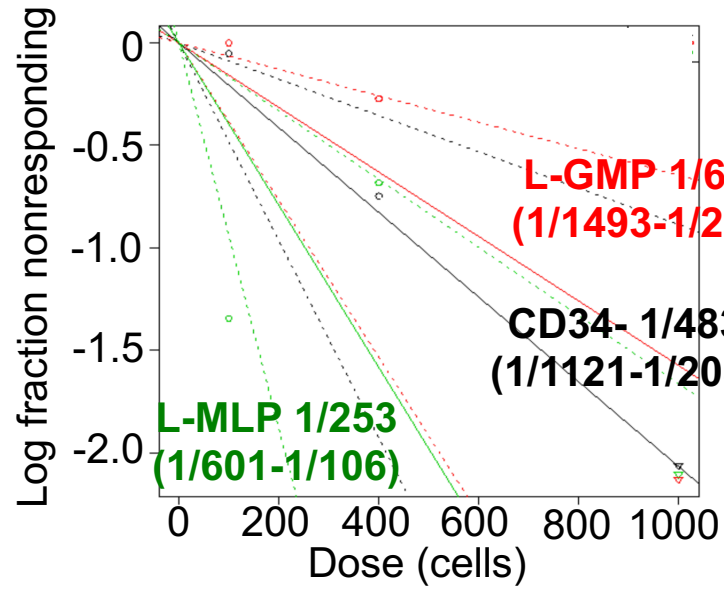

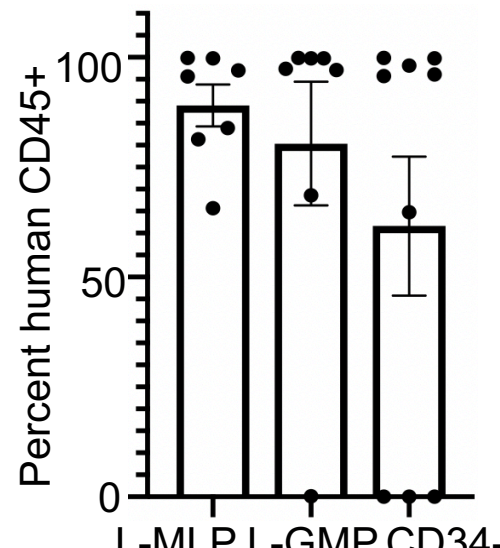

Source cell

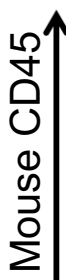

L-MLP

L-GMP

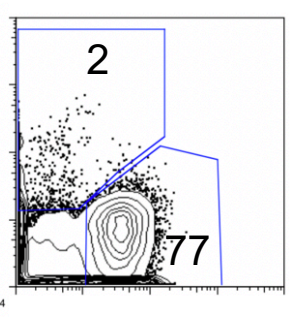
CD34-
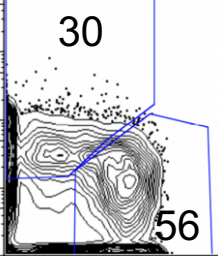

Human CD45 
Figure S3 (continued)

G

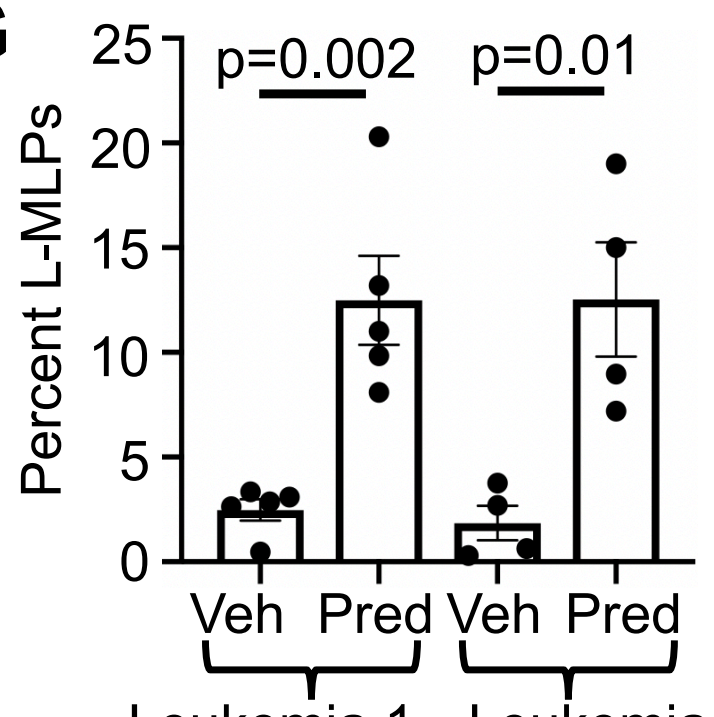

Leukemia 1 Leukemia 2

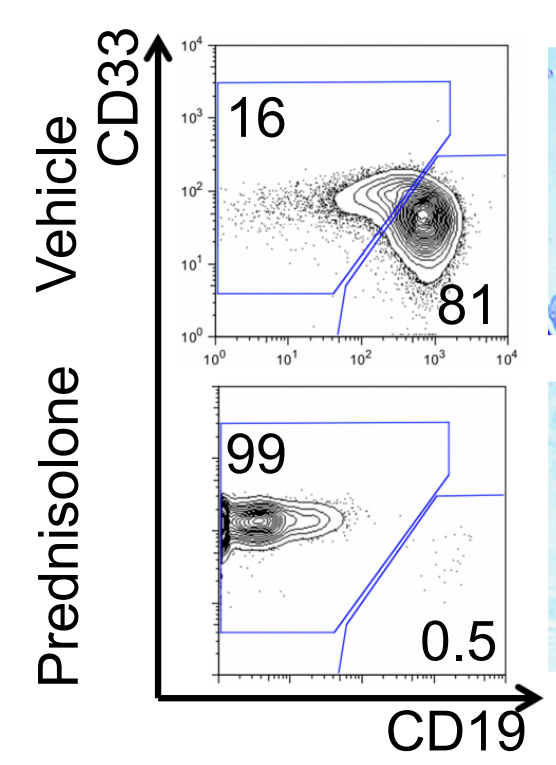

$\mathrm{H}$
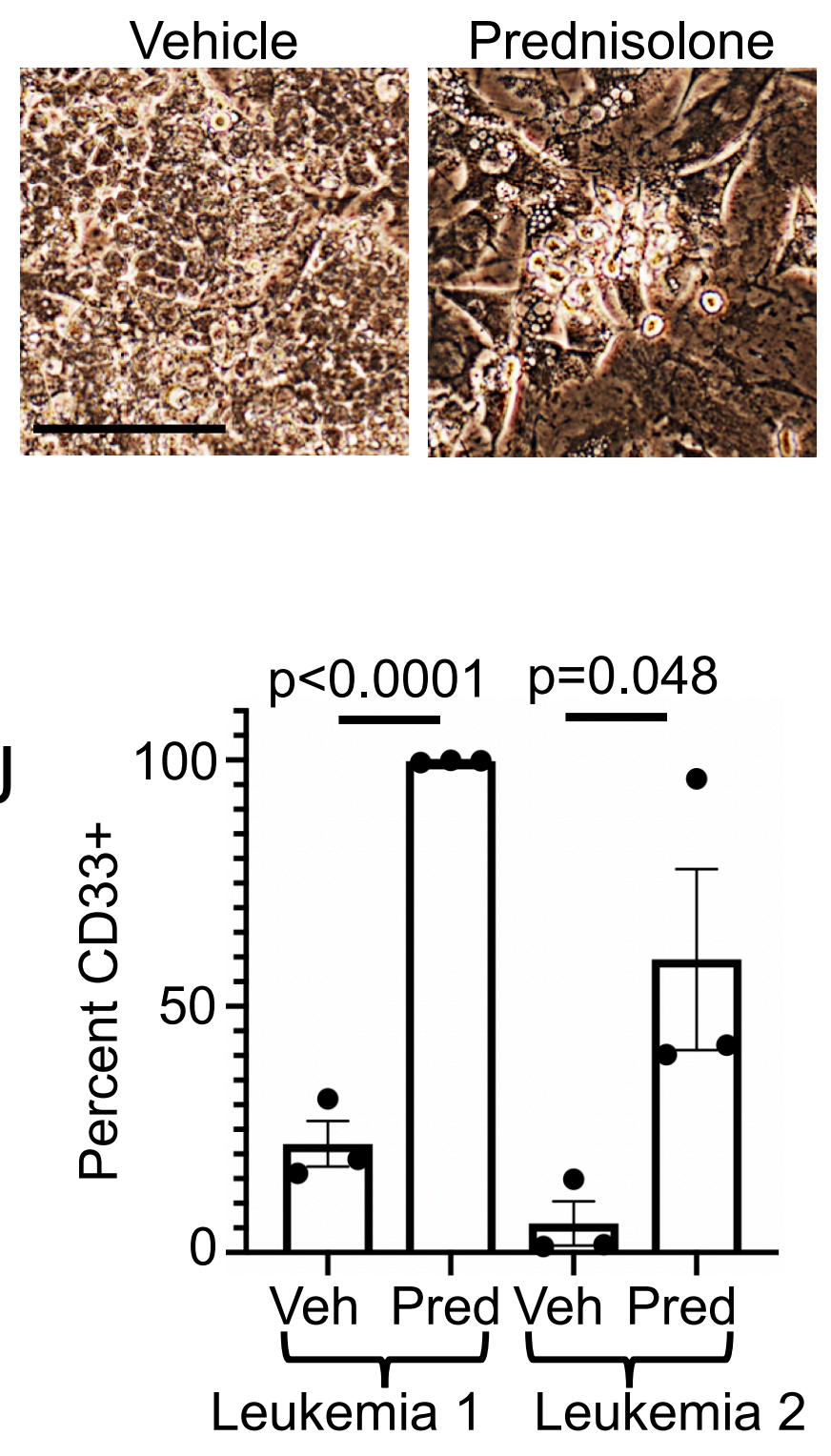
$A \quad \stackrel{m}{\infty}$ L-MLP L-GMP CD34-

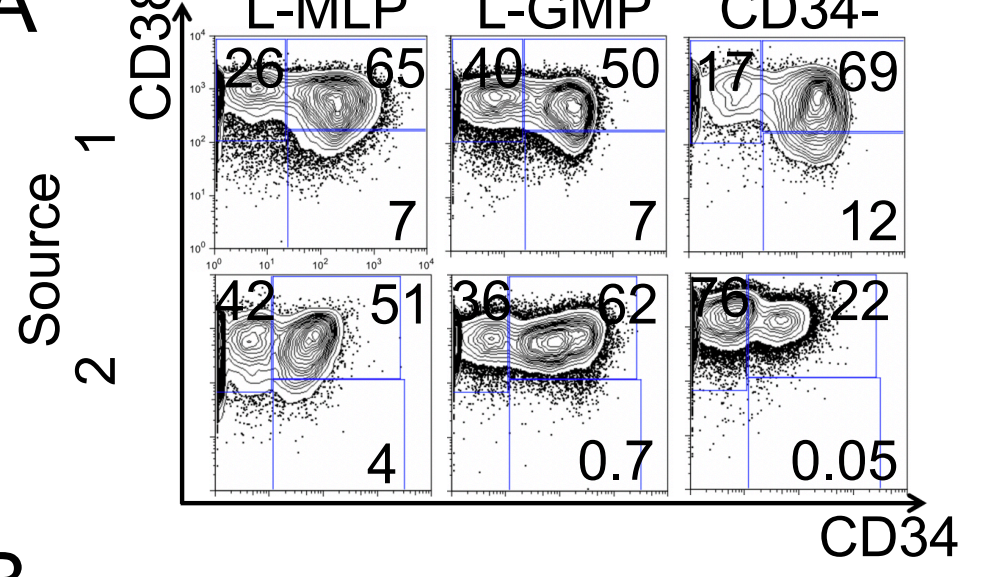

Figure S4

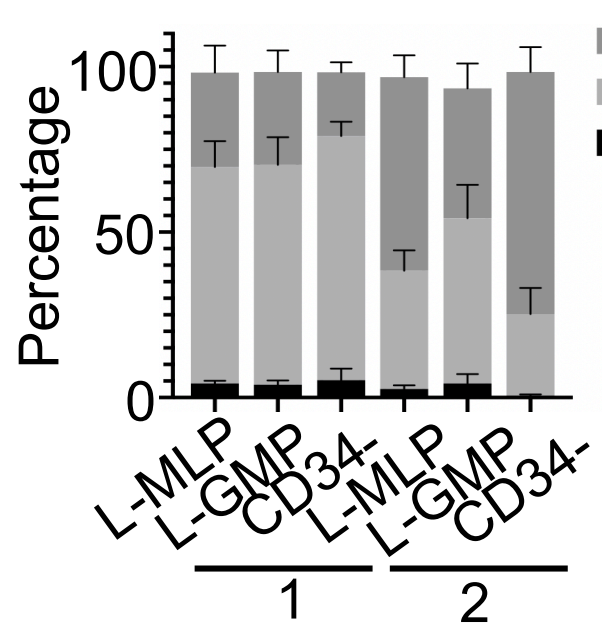
CD34L-GMP

- L-MLP

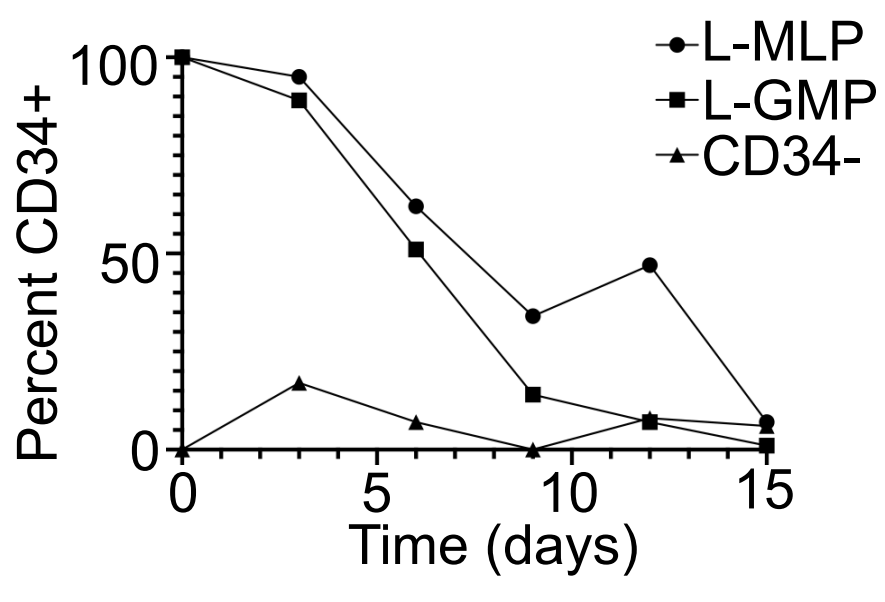

CD34

B
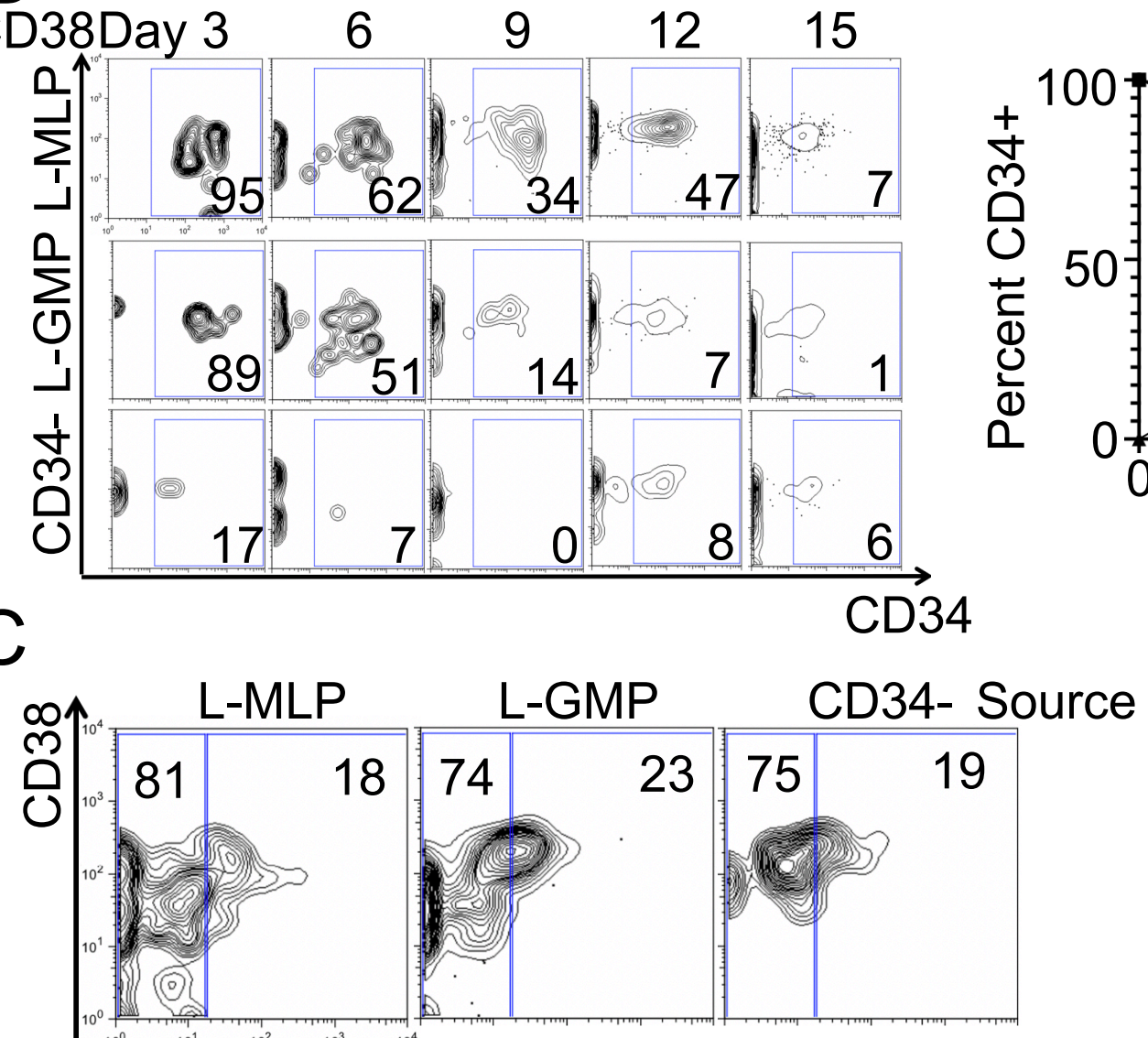

D

CD34

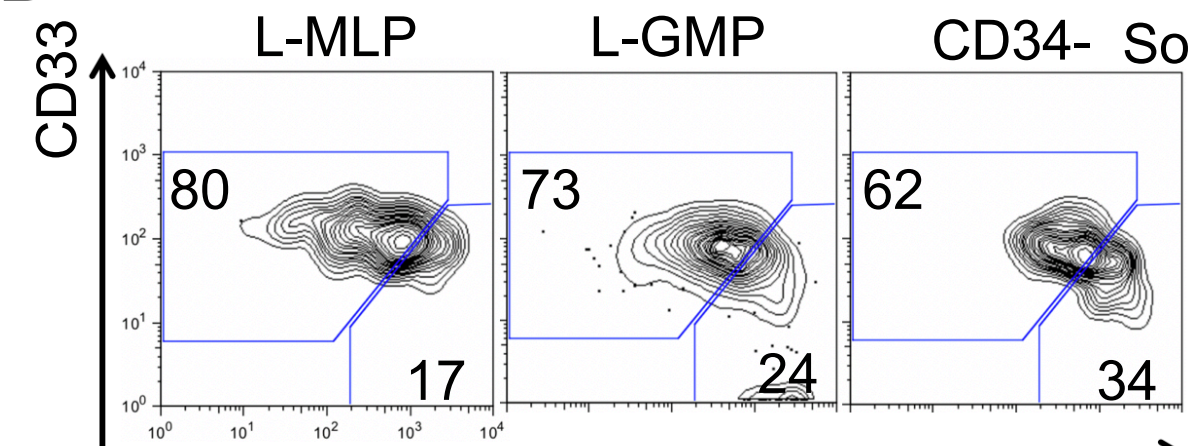

$\overrightarrow{C D} 19$
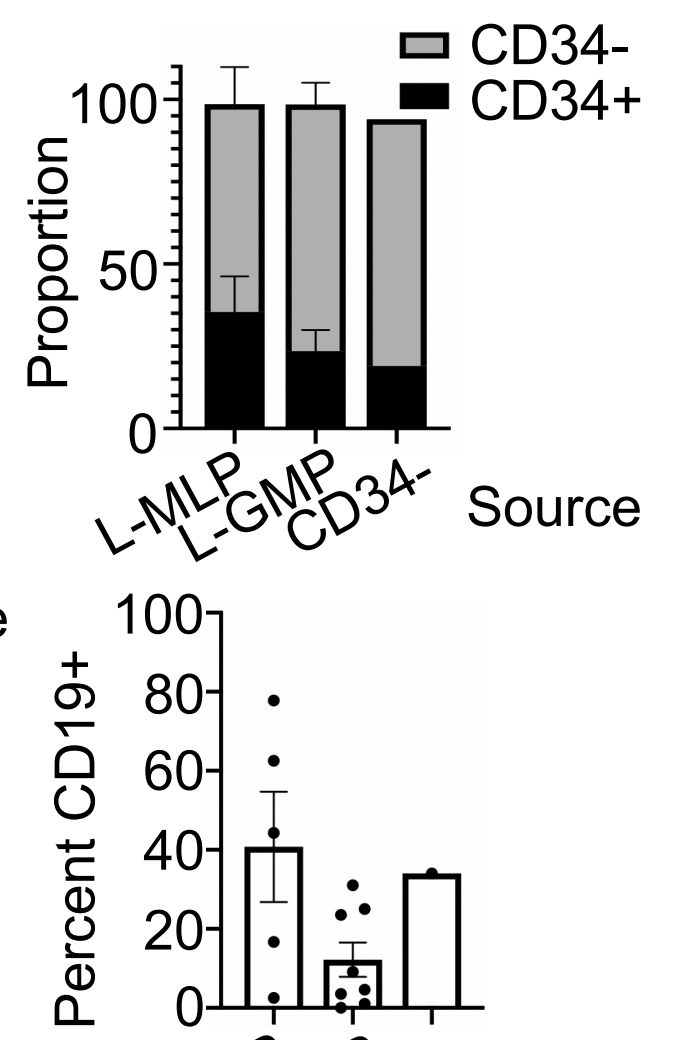

LML 